\title{
Article
}

\section{Spatial and kinematic segregation in star-cluster merger remnants}

Cole, David R., Debattista, Victor P, Varri, Anna-Lisa, Adam, Markus and Seth, Anil C.

Available at http://clok.uclan.ac.uk/16875/

Cole, David R., Debattista, Victor P ORCID: 0000-0001-7902-0116, Varri, AnnaLisa, Adam, Markus and Seth, Anil C. (2017) Spatial and kinematic segregation in star-cluster merger remnants. Monthly Notices of the Royal Astronomical Society, 466 (3). pp. 2895-2909. ISSN 0035-8711

It is advisable to refer to the publisher's version if you intend to cite from the work. http://dx.doi.org/10.1093/mnras/stw3325

For more information about UCLan's research in this area go to http://www.uclan.ac.uk/researchgroups/ and search for < name of research Group $>$.

For information about Research generally at UCLan please go to http://www.uclan.ac.uk/research/

All outputs in CLoK are protected by Intellectual Property Rights law, including Copyright law. Copyright, IPR and Moral Rights for the works on this site are retained by the individual authors and/or other copyright owners. Terms and conditions for use of this material are defined in the policies page.

\section{CLoK}

Central Lancashire online Knowledge www.clok.uclan.ac.uk

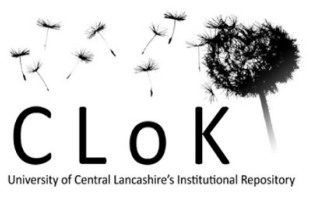




\title{
Spatial and kinematic segregation in star-cluster merger remnants
}

\author{
David R. Cole, ${ }^{1,2 \star}$ Victor P. Debattista, ${ }^{2}$ Anna-Lisa Varri, ${ }^{3}$ Markus Adam ${ }^{4}$ \\ and Anil C. Seth ${ }^{5}$ \\ ${ }^{1}$ Rudolf Peierls Centre for Theoretical Physics, Keble Road, Oxford OX1 3NP, UK \\ ${ }^{2}$ Jeremiah Horrocks Institute, University of Central Lancashire, Preston PR1 2HE, UK \\ ${ }^{3}$ School of Mathematics and Maxwell Institute for Mathematical Sciences, University of Edinburgh, Edinburgh EH9 3JZ, UK \\ ${ }^{4}$ SAP SE, Dietmar-Hopp-Allee 16, D-69190 Walldorf, Germany \\ ${ }^{5}$ Department of Physics and Astronomy, University of Utah, Salt Lake City, UT 84112, USA
}

Accepted 2016 December 9. Received 2016 December 9; in original form 2016 May 3

\begin{abstract}
Globular clusters that exhibit chemical and dynamical complexity have been suggested to be the stripped nuclei of dwarf galaxies(e.g. M54, $\omega$ Cen). We use $N$-body simulations of nuclear star clusters forming via the mergers of star clusters to explore the persistence of substructure in the phase space. We find that the observed level of differentiation is difficult to reconcile with the observed if nuclear clusters form wholly out of the mergers of star clusters. Only the star clusters that merged most recently retain sufficiently distinct density and kinematics to be distinguishable from the rest of the nuclear cluster. In situ star formation must therefore be included to explain the observed properties of nuclear star clusters, in good agreement with previous results.
\end{abstract}

Key words: galaxies: bulges-galaxies: evolution-galaxies: kinematics and dynamicsgalaxies: nuclei-galaxies: structure.

\section{INTRODUCTION}

High-resolution Hubble Space Telescope (HST) observations have shown that many low-to-intermediate-mass galaxies across the Hubble sequence contain a dense star cluster (SC) at their centre, a nuclear star cluster (NSC; Carollo et al. 1997; Böker et al. 2002; Côté et al. 2006; Turner et al. 2012).

NSCs in late-type galaxies are found to have complex star formation histories with mean luminosity-weighted ages ranging from 10 Myr to 10 Gyr (Rossa et al. 2006). Observations frequently show that the star formation is bursty, recurring on a time-scale of the order of $100 \mathrm{Myr}$ with the most recent episodes in the last $100 \mathrm{Myr}$ (Walcher et al. 2005, 2006). One example is the NSC in M33 that had periods of star formation $40 \mathrm{Myr}$ and $1 \mathrm{Gyr}$ ago (Long et al. 2002). Georgiev \& Böker (2014) studied 228 late-type galaxies and found that recent star formation is common and their stellar populations had a range of ages. Carson et al. (2015) found increasing roundness at longer wavelengths in HST Wide Field Camera images of the 10 brightest and nearest NSCs. They inferred that the NSCs contained discs with younger stellar populations. Colourcolour diagrams for most of these NSCs also show evidence for two populations, a younger one of the order of a few hundred Myr old and an older one more than a Gyr old. Pfuhl et al. (2011) studied the Milky Way's NSC and found that $\sim 80$ per cent of its stars are more

\footnotetext{
^E-mail: david.cole@physics.ox.ac.uk
}

than 5 Gyr old but there was a deep minimum in star formation 1-2 Gyr ago followed by an increase in star formation in the last few hundred Myr. NSCs in late-type galaxies are often made of an older spheroidal component with a younger, bluer disc embedded in it, with the disc approximately aligned with the plane of the main galactic disc (Seth et al. 2006, 2008). The NSC in NGC 4244 has such a structure and the stars in the disc are less than $100 \mathrm{Myr}$ old. Integral field spectroscopy indicates that the disc is rotating in the same sense as the main galactic disc and is misaligned by only $\sim 15^{\circ}$. The NSC in the elliptical galaxy FCC 277 also has the spheroid+disc structure with stars overall younger than those in the main galaxy (Lyubenova et al. 2013).

Two principal formation mechanisms have been proposed to explain the formation of NSCs: the merging of globular clusters (GCs), and in situ star formation. In the GC merger scenario, the GC orbits decay due to dynamical friction and then they merge at the centre of galaxies (Tremaine, Ostriker \& Spitzer 1975; Capuzzo-Dolcetta 1993; Miocchi et al. 2006; Capuzzo-Dolcetta \& Miocchi 2008a,b; Antonini et al. 2012; Antonini 2013, 2014; Arca-Sedda \& Capuzzo-Dolcetta 2014; Gnedin, Ostriker \& Tremaine 2014; Arca-Sedda et al. 2015). In situ star formation could occur due to a variety of mechanisms but would require a process whereby gas is driven to the nuclear regions of galaxies (Milosavljević 2004; Bekki 2007; Antonini, Barausse \& Silk 2015). These include the action of re-ionization epoch radiation fields (Cen 2001) and compressive tidal fields (Emsellem \& van de Ven 2008). Georgiev \& Böker (2014) found that the half-light 
radius, $r_{\text {eff }}$, of their sample of NSCs increases with wavelength and argue that this could be explained if NSCs form from gas that falls to the centre and forms stars, meaning that younger populations will be more centrally concentrated than older ones. The most direct evidence for the need of in situ star formation comes from modelling the kinematic data for the NSC in NGC 4244. Simulations by Hartmann et al. (2011, see also De Lorenzi et al. 2013) find that though the GC merger scenario can reproduce many of the density and kinematic properties of NSCs, mergers give rise to a central peak in $v_{\mathrm{rms}}=\sqrt{\sigma_{\mathrm{los}}^{2}+v_{\mathrm{los}}^{2}}$, which is not observed in the data. Here, $v_{\text {los }}$ is the observed mean stellar velocity and $\sigma_{\text {los }}$ is the mean stellar velocity dispersion. Based on this, they conclude that less than 50 per cent of the mass of the NSC could have been assembled from the mergers of GCs, with the majority due to in situ star formation.

Turning our attention to GCs, the interpretative paradigm for their formation and dynamical evolution is also puzzling. At one time, the Milky Way's GCs were thought to consist of a single stellar population, but the availability, over the past decade or so, of highquality and homogeneous photometric and spectroscopic data sets has revealed a much more complex picture of the star formation history of this class of stellar systems. In particular, there is now clear evidence that most Galactic GCs exhibit light element abundance patterns and colour-magnitude diagram morphology indicative of the existence of multiple stellar populations (see e.g. Gratton, Carretta \& Bragaglia 2012; Piotto et al. 2015). A number of possible scenarios have been proposed to provide an interpretation of such a ubiquitous and puzzling phenomenon, often invoking the presence of two (or more) generations of stellar populations, with several different possible sources for the gas out of which second population stars form. These sources include rapidly rotating massive stars, massive binary stars, and intermediate-mass asymptotic giant branch (AGB) stars (see e.g. Ventura et al. 2001; Prantzos \& Charbonnel 2006; D'Ercole et al. 2008, 2010, 2012; de Mink et al. 2009). Alternative scenarios further elaborate on the role of the ejecta from massive interacting binaries, in the context of the formation of circumstellar discs of young, low-mass stars (Bastian et al. 2013), as a possible origin for the observed abundance anomalies.

One crucial insight into this problem may arise from the investigation of the structural (see e.g. Vesperini et al. 2013) and kinematical properties (see e.g. Mastrobuono-Battisti \& Perets 2013; Hénault-Brunet et al. 2015) of multiple stellar populations. Lardo et al. (2011) have studied nine Galactic GCs with Sloan Digital Sky Survey (SDSS) data, and found that there is a statistically significant spread in $u-g$ colour, corresponding to variations in the abundances of light elements, with the redder stars being more centrally concentrated than the bluer ones. They concluded that there are distinct populations that have different radial distributions. From the kinematic perspective, Richer et al. (2013) analysed the proper motions of main-sequence stars in 47 Tuc by dividing them into four colour bands, assuming that the colour bands represent stars with different chemical composition. They found that the mainsequence stars in 47 Tuc have anisotropic proper motions, and that such a feature is correlated with their colours. They also found that the bluest stars are also the most centrally concentrated, confirming that, also in the case of 47 Tuc, different stellar populations can be distinguished by their spatial distribution. More recently, Bellini et al. (2015) have studied the kinematic properties of multiple populations in NGC 2808 on the basis of high-precision HST proper-motion measurements, and found that the helium-enriched populations are more radially anisotropic. Generally these stud- ies identify stellar populations photometrically or spectroscopically and then analyse either their spatial or kinematic distribution. Sometimes, this includes examining population kinematics in a given radial range, but, in general, observational studies have not yet examined the joint stellar distribution in both space and velocity. All aspects of the formation, chemistry, and dynamical evolution of GCs are currently intensely debated (see e.g. Bastian 2015; Renzini et al. 2015; D'Antona et al. 2016), and only the synergy between state-of-the-art photometric (especially the HST UV Legacy Survey of Galactic GCs, presented by Piotto et al. 2015), spectroscopic (see Carretta 2015; Lardo et al. 2015), and proper motion (from HST and Gaia, e.g. see Watkins et al. 2015; Pancino, Bellazzini \& Marinoni 2013, respectively) information will allow us to address many of these open questions.

One additional (and older) puzzle is the existence of GCs with significant variations in their heavy elements abundances. In this respect, evolutionary scenarios that include one or more merger events often have been formulated as a possible formation channel of these 'multimetallic clusters' (van den Bergh 1996; Catelan 1997; Lee et al. 1999; Carretta et al. 2010a, 2011; Bekki \& Yong 2012; Amaro-Seoane et al. 2013). The scenarios in this class have often been considered rather unlikely in the Galactic environment, but not unrealistic in other settings, such as in interacting galaxies (e.g. the Antennae) or in the core of a dwarf galaxy (e.g. Sagittarius). In particular, it has been envisaged that GCs may come close and merge due to galaxy interactions or where GCs have fallen to the centre of the host system due to dynamical friction. Amaro-Seoane et al. (2013) investigated this process using $N$-body simulations and found that the radial distribution of different populations are similar to those in multimetallic GCs. In particular, they found that the distribution of stellar populations in their dynamical models had some resemblance to the observed distribution in $\omega$ Cen. However, Catelan (1997) found that a merger of two GCs would produce a red giant branch with bimodal colours and no such bimodality had been seen in Galactic GCs, which led to the conclusion that they are unlikely to be formed by mergers. Ferraro et al. (2009) have found that Terzan 5 shows bimodality in the red clump and red giant branch.

Interestingly, Galactic GCs that are characterized by anomalous metallicity distributions tend to be also particularly massive. These two aspects, coupled with additional signatures of dynamical complexity, have often been interpreted as possible indications that these stellar systems may be remnants of dwarf galaxies, which have been tidally stripped by the potential of the Milky Way (e.g. van den Bergh 1996; Bekki \& Norris 2006). In this context, it has also been speculated that these objects are actually able to retain fast supernovae ejecta (hence the spread in heavy elements), as they were much more massive at their birth, further supporting the possibility of identifying them as nuclei of disrupted dwarf galaxies. Notable cases, as characterized by a very wide or even multimodal metallicity distribution, include $\omega$ Cen (Lee et al. 1999; Bekki \& Norris 2006; Carretta et al. 2010b), M54 (Sarajedini \& Layden 1995; Siegel et al. 2007; Carretta et al. 2010a), and Terzan 5 (Ferraro et al. 2009; Origlia et al. 2011; Massari et al. 2014).

Significant intrinsic iron spreads have been measured also in M22 (Da Costa et al. 2009; Marino et al. 2009, 2011), M2 (Yong et al. 2014), NGC 1851 (Carretta et al. 2010a, 2011; Bekki \& Yong 2012), and NGC 5286 (Marino et al. 2015, see their table 10). In this context, it should also be emphasized that the analysis of GCs identified as having an intrinsic Fe spread deserves particular care, especially with respect to non-local thermodynamical equilibrium effects driven by overionization mechanisms in the atmosphere of 
Table 1. Properties of $\omega$ Cen, 47 Tuc, NGC 1851, M54, M22, and Terzan 5 from Harris (1996). Although 47 Tuc does not show any evidence of enhancement or spread in its iron abundance (see Marino et al. 2016), we include it in this list in light of the estimated total mass, its complex light elements abundance patterns (e.g. see Cordero et al. 2014; Kučinskas, Dobrovolskas \& Bonifacio 2014), and its rich internal dynamics (e.g. see Bianchini et al. 2013; Richer et al. 2013).

\begin{tabular}{lcccc}
\hline Cluster & $\begin{array}{c}\text { Mass } \\
\times\left(10^{6} \mathrm{M}_{\odot}\right)\end{array}$ & $\begin{array}{c}\text { Absolute } \\
\text { visual mag. }\end{array}$ & $\begin{array}{c}\text { Half-mass } \\
\text { radius } \\
(\mathrm{pc})\end{array}$ & {$[\mathrm{Fe} / \mathrm{H}]$} \\
\hline$\omega$ Cen & $2-5^{a}$ & -10.24 & 6.20 & -1.62 \\
47 Tuc & $0.7-1.45^{b, c}$ & -9.37 & 3.49 & -0.76 \\
NGC 1851 & $0.561^{c}$ & -8.35 & 1.85 & -1.26 \\
M54 & $1.45^{c}$ & -9.96 & 3.76 & -1.59 \\
M22 & $0.536^{c}$ & -8.45 & 3.03 & -1.64 \\
Terzan 5 & $\sim 2^{d}$ & -7.86 & 1.93 & -0.28
\end{tabular}

${ }^{a}$ Meylan et al. (1995); van de Ven et al. (2006); D’Souza \& Rix (2013).

${ }^{b}$ Marks \& Kroupa (2010).

${ }^{c}$ Gnedin \& Ostriker (1997).

${ }^{d}$ Lanzoni et al. (2010).

AGB stars, which may lead to spurious metallicity assessments (e.g. see Lapenna et al. 2014; Mucciarelli et al. 2015).

The half-mass radii and masses of many clusters listed above are consistent with those of NSCs (see Table 1, and also fig. 3 of Walcher et al. 2005). In particular, $\omega$ Cen has been often identified as a very peculiar SC, not just in consideration of its chemical complexity, but also for a number of interesting kinematical and dynamical features (such as the strong internal rotation; Sollima et al. 2009; Bianchini et al. 2013), especially the possibility of the presence of a central, dynamically decoupled substructure, as revealed by the Schwarzschild model of van de Ven et al. (2006). M54 is thought to be the NSC of the Sagittarius dSph and, as such, to be in the process of being stripped by the Galactic potential (but Siegel et al. 2011, find that M54 may be $2 \mathrm{kpc}$ in the foreground of the centre of the Sagittarius dSph; although, this would require an unusual alignment). There is also evidence for the presence of an intermediate-mass black hole in both M54 and $\omega$ Cen, which also favours them being stripped NSCs (Noyola, Gebhardt \& Bergmann 2008; Ibata et al. 2009; Miocchi 2010; Wrobel, Greene \& Ho 2011); although, especially in the case of $\omega$ Cen, this issue is still highly debated (Anderson \& van der Marel 2010; van der Marel \& Anderson 2010).

Inspired by the new recognition of chemical and dynamical complexity that seems to characterize these stellar systems, possibly at the interface between GCs and NSCs, we wish to perform an investigation of a number of structural, kinematical, and phase-space properties of the products of numerical experiments of GCs mergers, as a possible formation scenario of NSCs. In particular, we wish to assess the persistence of any structural and kinematical distinction between the different components, associated with the original GCs, within the stellar system resulting from the merger process. An analysis, devoted to the exploration of the spatial and age differences among different mass components, has recently been presented by Perets \& Mastrobuono-Battisti (2014); in the present investigation, we wish to devote our attention in particular to the kinematical and dynamical properties.

To test whether different components can be distinguished spatially and kinematically in the case of GC merging, we have studied two simulations of this process. In simulation S1, a number of GCs merge to form a larger object, while in simulation $\mathrm{S} 2$ we start with a pre-existing SC, and then add several GCs, one at a time, to merge to the central object. We then study the spatial distributions and the kinematics of the stars originating in different GCs, and investigate how well mixed they are. The article is organized as follows: Section 2 describes the simulation methods, Section 3 describes the resultant SCs for both simulations, and finally Section 4 presents a discussion of our conclusions.

\section{THE SIMULATIONS}

Our $N$-body simulations were run using the efficient parallel tree code PKDGRAV (Stadel 2001) suitable for studying collisionless dynamics. Collisional dynamics will become important over periods comparable with the relaxation time that is $\sim 10 \mathrm{Gyr}$ for our merger remnants, significantly longer than the time-scales of our simulations. The effect of collisional dynamics would be to erase further any differences in spatial and kinematic properties of stellar populations implying that it would become harder to distinguish stellar populations as time went on. In this sense, our results represent the optimum chance of identifying stellar populations by their spatial and velocity distributions. Simulation S1 has not been described previously and we provide a description here. Simulation S2 was described by Hartmann et al. (2011) and we only provide a brief description of it here. We have rescaled each simulation a posteriori, which we are at liberty to do as they are collisionless, so that the final merger remnant has similar mass and size to $\Omega$ Cen. See Table 1 for the observed values of the mass and half-mass radius of $\Omega$ Cen and Tables 2 and 3 for the mass and half-mass radii for our simulations. All the data and figures are shown in units that reflect this rescaling.

Both simulations S1 and S2 evolve within a bulge. The bulge model has a Hernquist (1990) profile:

$\rho(r)=\frac{a M_{\mathrm{b}}}{2 \pi r(r+a)^{3}}$,

where the mass is $1.25 \times 10^{8} \mathrm{~m}_{\min }$, where $m_{\min }$ is the minimum particle mass and the scale radius is $a$ (for the actual mass and scale radius in our simulations see below). The density is truncated smoothly in energy space at $r>15 a$ (Sellwood \& Debattista 2009). The bulge is made up of $3.5 \times 10^{6}$ particles with masses ranging

Table 2. Properties of the merger remnant in simulation S1 at several times. $N_{\mathrm{GC}}$ is the number of GCs that have merged to that point.

\begin{tabular}{lccc}
\hline $\begin{array}{l}\text { Time } \\
(\mathrm{Myr})\end{array}$ & $\begin{array}{c}\text { Mass } \\
\times\left(10^{6} \mathrm{M}_{\odot}\right)\end{array}$ & $\begin{array}{c}\text { Half-mass radius } \\
(\mathrm{pc})\end{array}$ & $N_{\mathrm{GC}}$ \\
\hline 140 & 1.83 & 2.42 & 3 \\
280 & 3.03 & 4.26 & 5 \\
420 & 3.05 & 4.36 & 5 \\
560 & 3.05 & 4.36 & 5 \\
1090 & 3.6 & 6.2 & 6 \\
\hline
\end{tabular}

Table 3. Properties of the merger remnant in simulation S2 at three stages of its evolution.

\begin{tabular}{lccc}
\hline $\begin{array}{l}\text { Time } \\
(\mathrm{Myr})\end{array}$ & $\begin{array}{c}\text { Mass } \\
\times\left(10^{5} \mathrm{M}_{\odot}\right)\end{array}$ & $\begin{array}{c}\text { Half-mass radius } \\
(\mathrm{pc})\end{array}$ & $N_{\mathrm{GC}}$ \\
\hline 115 & 16.22 & 2.9 & 10 \\
220 & 28.39 & 6.2 & 20 \\
300 & 36.89 & 6.95 & 27 \\
\hline
\end{tabular}


from $m_{\min }$ at the centre to $10^{4} m_{\min }$ further out giving increased mass resolution within $0.1 a$ (Sellwood 2008). The bulge has no strong instabilities meaning that the distribution of particles remains unchanged on time-scales up to a Gyr, more than adequate to model multiple accretions of GCs. It should be emphasized that the mass resolution in the inner $0.02 a$ of the bulge model is very high and very stable as shown by fig. 8 of Hartmann et al. (2011), as required for proper dynamical modelling of the accretion of our SCs. At least 60 percent of the mass of the bulge in the inner $0.02 a$ is made up of particles with mass $\leq 2 m_{\min }$ throughout the simulation and there is no substantial change in the distribution of particle masses.

In simulation S1, there was no initial structure at the centre of the bulge; SCs were placed on orbits close to the centre, which decayed due to dynamical friction, falling to the centre, where 6 of initially 10 SCs merged to form a nuclear remnant. In order to approximate $\Omega$ Cen, the bulge model has $m_{\min }=120 \mathrm{M}_{\odot}$ and $a=1.0 \mathrm{kpc}$. The SC models then have particles of equal mass $\left(15 \mathrm{M}_{\odot}\right)$ and softening $(\epsilon=0.08 \mathrm{pc})$. Their concentration $c=0.12$ is defined as $c=\log \left(R_{\mathrm{eff}} / R_{\mathrm{c}}\right)$, where $R_{\mathrm{eff}}=0.65 \mathrm{pc}$ is the half-mass radius (effective radius) and $R_{\mathrm{c}}$ is the core radius, where the surface density drops to half of the central. The orbits of the $10 \mathrm{SCs}$ were found by selecting particles of the bulge with high angular momentum within a radius of $60 \mathrm{pc}$. We placed the SCs at the same position and with the same velocities as these bulge particles. Afterwards we rotated the SC system by $180^{\circ}$ around the centre to avoid the SCs being coincident with the chosen particles. The orbits of our SCs had random initial orientations and so were not coplanar. The orientation of the angular momentum that the SCs contribute to the merger remnant is therefore not aligned. The initial distances of the SCs from the centre range from 30 to $60 \mathrm{pc}$, with velocities in the range of $40-270 \mathrm{~km} \mathrm{~s}^{-1}$. The model SCs have a mass of $6 \times 10^{5} \mathrm{M}_{\odot}$, comparable to GCs found in the Milky Way and the Local Group (Figer, McLean \& Morris 1999; Figer et al. 2002; Mackey \& Gilmore 2003; McLaughlin \& van der Marel 2005). The SC model is an isotropic distribution function of a lowered polytrope with index $n=2$ :

$f(x, v) \propto[-2 E(x, v)]^{1 / 2}-\left[-2 E_{\max }\right]^{1 / 2}$.

An iterative process is used to produce equilibrium models (Debattista \& Sellwood 2000).

Simulation S2 is the same as run A1 of Hartmann et al. (2011), which was also studied by Portaluri et al. (2013, and was also rescaled). $\Omega$ Cen is approximated when the SC models have particles of equal mass $\left(3 \mathrm{M}_{\odot}\right)$ and equal softening $(\epsilon=0.04 \mathrm{pc})$. The concentration $c=0.16$ and $R_{\mathrm{eff}}=0.34 \mathrm{pc}$. This model is also comparable to massive young SCs in the Milky Way and the Local Group (Figer et al. 1999, 2002; Mackey \& Gilmore 2003; McLaughlin \& van der Marel 2005). We create an NSC for the SCs to accrete on to by letting a massive $\mathrm{SC}$ of similar profile and mass $=4 \times 10^{5} \mathrm{M}_{\odot}$, $c=0.12$, and $R_{\text {eff }}=0.66 \mathrm{pc}$, fall to the centre of the bulge. This $\mathrm{SC}$ was allowed to settle to the centre from a circular orbit at $38 \mathrm{pc}$, which takes $25 \mathrm{Myr}$, before we started the accretion of 27 GCs, starting them on circular orbits at a distance of $10 \mathrm{pc}$ from the centre. In total, the mass accreted corresponds to $\sim 8.1$ times the NSCs' initial mass. Each accreted GC is allowed to reach the centre of the cluster before a new GC is inserted. A single accretion on average requires $\sim 8 \mathrm{Myr}$ and the $27 \mathrm{GCs}$ are accreted in 300 Myr.

\section{RESULTS}

\subsection{Properties of the merger remnant in simulation S1}

\subsubsection{Final properties}

In this section, we examine the properties of the merger remnant formed in simulation $\mathrm{S} 1$ in terms of the stars that originated in each progenitor GC. The aim will be to see if we can distinguish the stars that originated in individual GCs as populations with distinct distributions and kinematics within the merger remnant.

Table 2 shows the mass and the half-mass radius, $R_{e}$, of the nuclear merger remnant at five times during the simulation. It should be noted that there have been mergers within the previous $70 \mathrm{Myr}$ at $t=140,280$, and $1090 \mathrm{Myr}$ but not at 420 and $560 \mathrm{Myr}$, which explains why there is very little change in $R_{e}$ between the latter times. Fig. 1 shows a map of the merger remnant's surface density within $4 R_{e}$ after $1.09 \mathrm{Gyr}$. It has a mass of $\sim 3.6 \times 10^{6} \mathrm{M}_{\odot}$ and a half-mass radius $R_{e} \sim 6.2 \mathrm{pc}$ with a mildly oblate shape.

Fig. 2 shows the density profiles and Fig. 3 shows the velocity dispersion profiles in cylindrical coordinates for the merger remnant
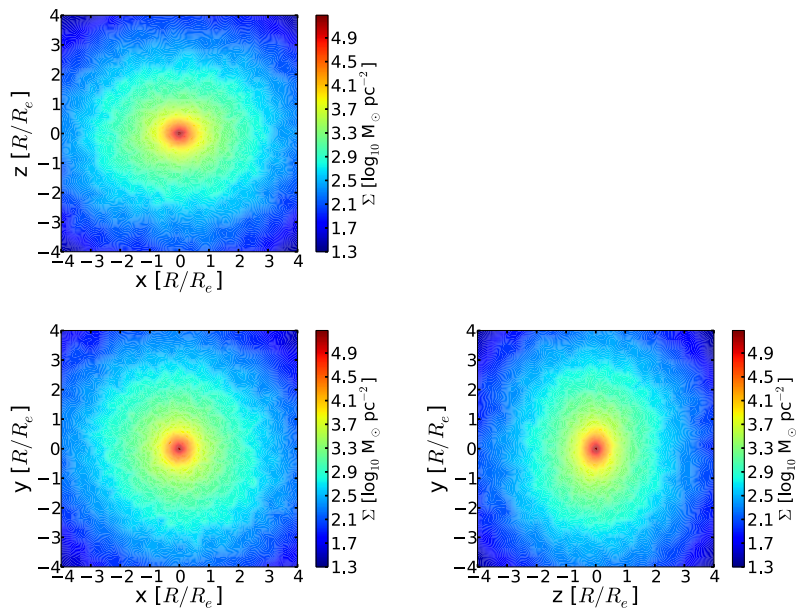

Figure 1. Surface-density map of the final merger remnant from simulation S1 showing three orthogonal projections. The system has been oriented so that the resulting angular momentum is about the $z$-axis.

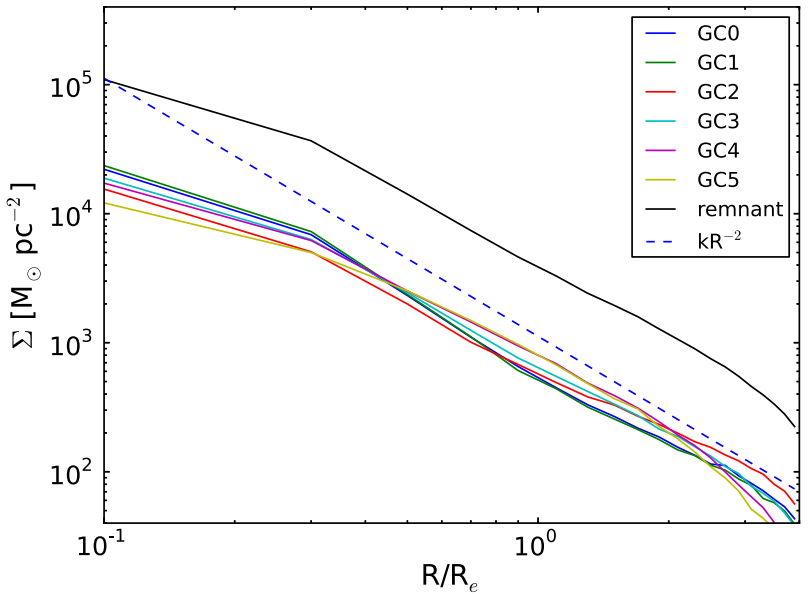

Figure 2. Cylindrical density profiles for the final merger remnant for simulation S1 showing the overall density and the densities contributed by stars originating in each progenitor GC. For comparison, the dashed (blue) line shows a power law with an exponent of -2 . 

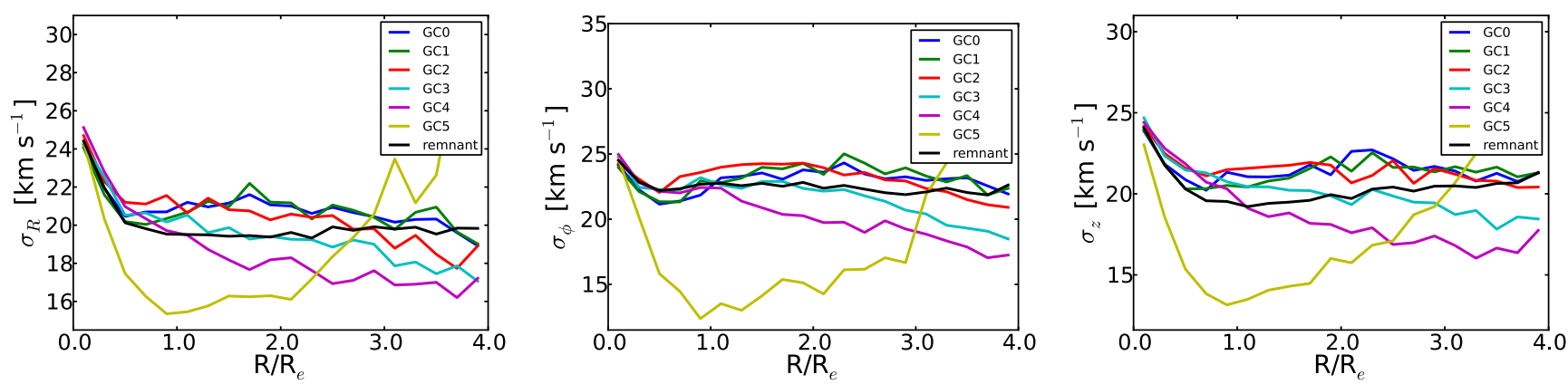

Figure 3. Velocity dispersions for the final merger remnant in simulation $\mathrm{S} 1$ at $1.09 \mathrm{Gyr}$ and for the stars originating in each progenitor GC. Left: $\sigma_{R}$; middle: $\sigma_{\phi} ;$ right: $\sigma_{z}$.

at the end of the simulation. We separate the stars by their progenitor GC, labelling the GCs in the order in which they merged, from GC0 to GC5. The contribution of each GC is distinguished as a different coloured line. It is apparent that it is difficult to visually identify different progenitor populations on the basis of the density profile and velocity dispersion, though GC5 looks distinguishable on the basis of its velocity dispersion. The final density distribution is very similar for the stars from all GCs. Even stars from the most recently merged GC5 have a very similar density profile to the stars originating in the other GCs. All velocity dispersions are similar for all the groups of stars originating in each GC except for GC5. GC5 shows the most significant deviations from the behaviour of all the other components; in particular, the radial profiles of the velocity dispersion tensor are systematically lower in the central to intermediate regions $\left(R<2 R_{e}\right)$, while they become comparable to the values of the other components in the outer parts $\left(R>2 R_{e}\right)$. The differences in dispersion between GCs $0,1,2$, and 3 are $\sim 3 \mathrm{~km} \mathrm{~s}^{-1}$, which would be hard to detect. GC4 has a maximum difference of $\sim 5 \mathrm{~km} \mathrm{~s}^{-1}$, which may be detectable, and the maximum difference of GC5's velocity dispersion from the mean is for $\sigma_{\phi}$ and is $\sim 8 \mathrm{~km} \mathrm{~s}^{-1}$ at $R \sim R_{e}$.

\subsubsection{Merger remnant evolution in simulation S1}

We now consider whether it is possible to distinguish different progenitor populations at earlier times in the simulation. Fig. 4 shows the merger remnant density profile at $140 \mathrm{Myr}$ and at $280 \mathrm{Myr}$, times at which GCs have recently merged into the remnant. When $\mathrm{GC} 0$, GC1, and GC2 have merged, the merger remnant has a mass of $1.83 \times 10^{6} \mathrm{M}_{\odot}$ and a half-mass-radius of $\sim 2.42 \mathrm{pc}$. After GC3 and GC4 have also merged, the remnant's mass has increased to $3.03 \times 10^{6} \mathrm{M}_{\odot}$ and the half-mass radius to $\sim 4.26 \mathrm{pc}$. There is a noticeably larger difference in the density profiles of the most recently merged GCs, having a flatter central density. By the time five GCs have merged, the density profile for GC2, which was quite different from the rest when just three GCs had merged, has become close to the typical density profile of the other GCs. This evolution is due to violent relaxation caused by subsequent mergers (see Section 4). Examination of these density profiles in spherical coordinates shows similar profiles demonstrating that these plots are not affected by projection effects.

Fig. 5 shows the merger remnant velocity dispersion profiles within $4 R_{e}{ }^{1}$ after three GCs have merged and after five GCs have

\footnotetext{
${ }^{1}$ We choose this radius because Kučinskas et al. (2014) include stars out to $4 R_{e}$ in their work on 47 Tuc.
}
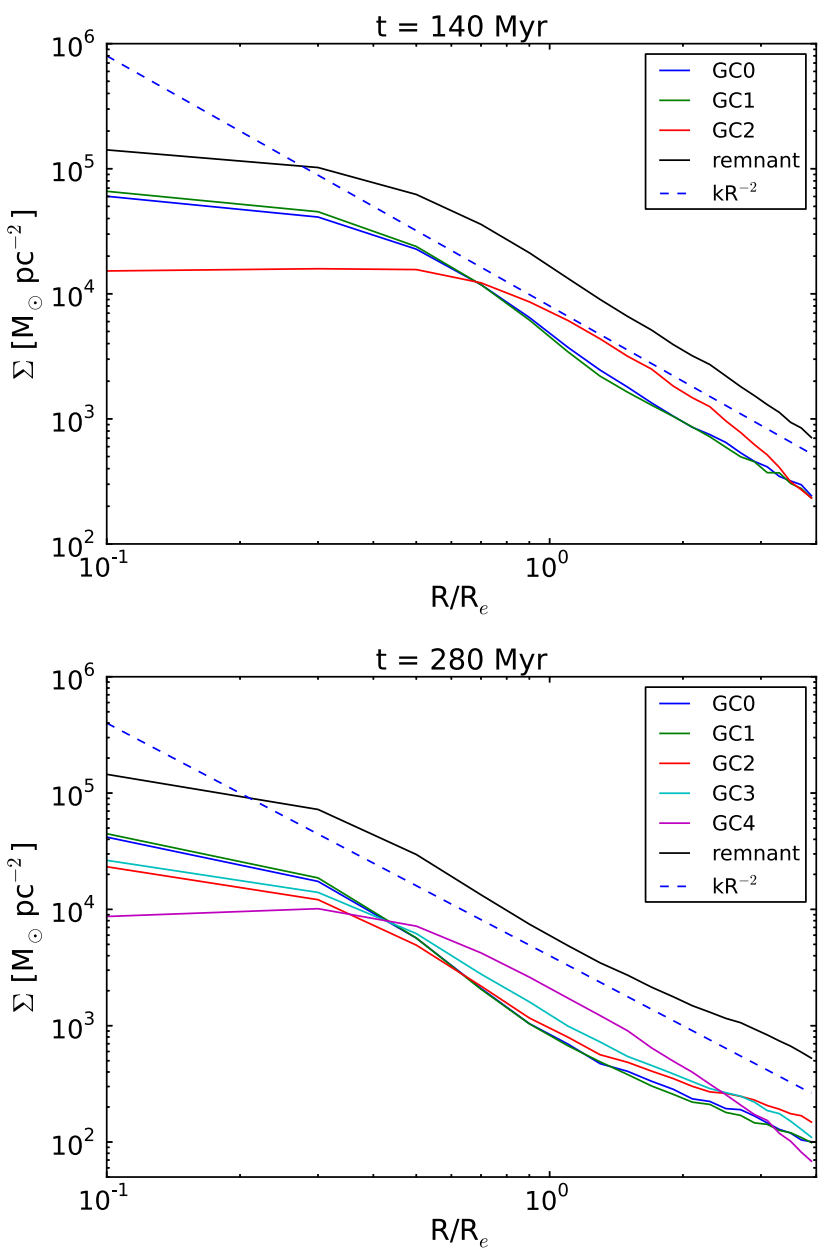

Figure 4. The density profile of the stars from individual GCs soon after a merger has occurred in simulation S1. Top: after $140 \mathrm{Myr}$, the first three GCs have merged with GC2 being the most recent. Bottom: after $280 \mathrm{Myr}, \mathrm{GC} 3$ and $\mathrm{GC} 4$ have now merged with GC4 being the most recent. For comparison, the dashed (blue) lines show a power-law relation with an exponent of -2 .

merged. These plots generally show the velocity dispersion profiles are similar in shape and magnitude for all stars originating in each GC. After three GCs have merged, the most recently merged GC2 shows the greatest deviation from the mean at $\lesssim 6 \mathrm{~km} \mathrm{~s}^{-1}$. After the merger of two additional GCs, it is again the most recently merged GC4 that shows lower velocity dispersions with a maximum 

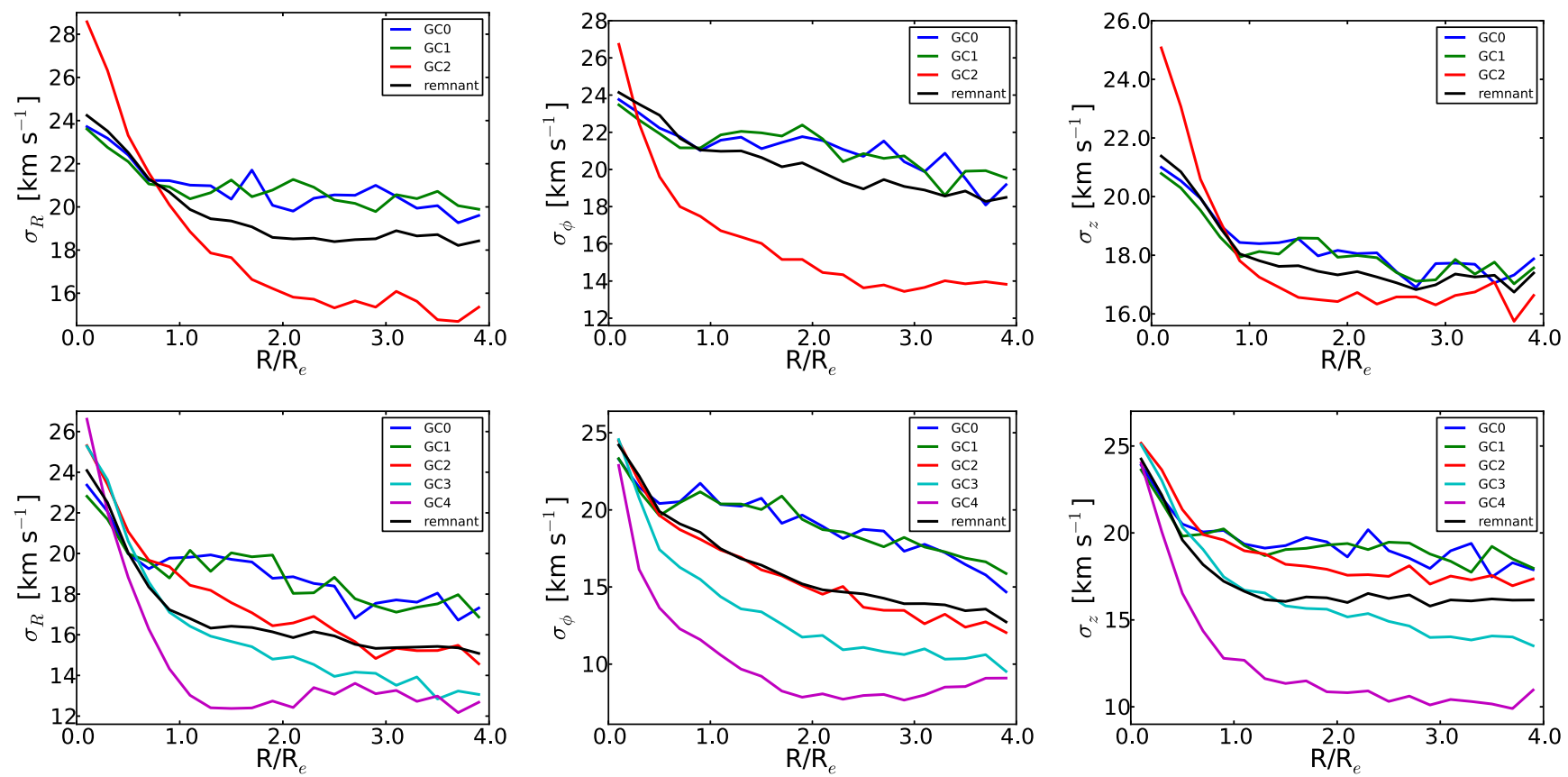

Figure 5. The velocity dispersion profile of the stars originating from individual GCs after three GCs have merged (top) and after five GCs have merged (bottom) in simulation $\mathrm{S} 1 . \sigma_{R}$ is shown on the left, $\sigma_{\phi}$ in the middle, and $\sigma_{z}$ on the right.

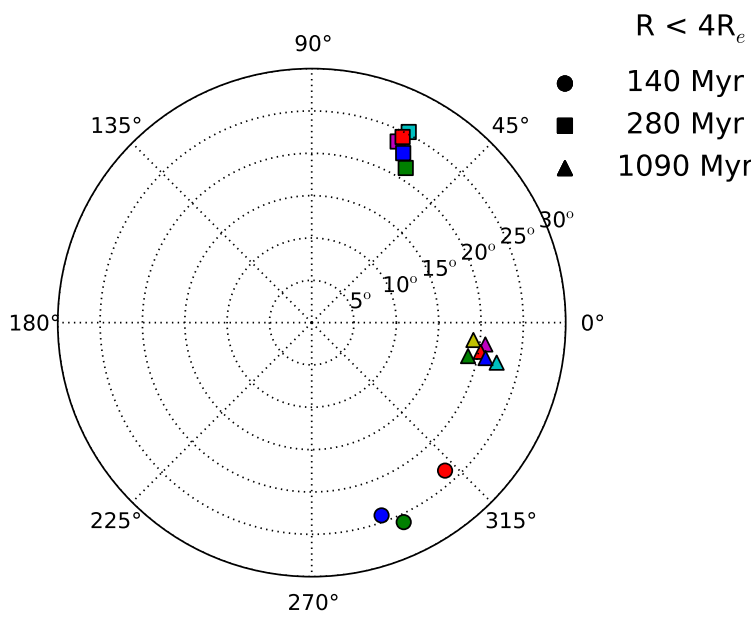

Figure 6. Briggs figure for the stars from different GCs in model 1. The direction of the angular momentum vector for each GC is indicated by the position of the symbol on the plot. Circles are values after three GCs have merged, squares after five GCs have merged, and triangles after six GCs have merged. The plot shows the values for stars inside of $4 R_{e}$. We use the same colour code as in other figures: GC0 - blue; GC1 - green; GC2 - red; GC3 - cyan; GC4 - magenta; and GC5 - yellow.

difference of $\sim 5 \mathrm{~km} \mathrm{~s}^{-1}$ from the mean. GC3 and GC4 are quite distinguishable here, indicating that mixing is less complete.

Fig. 6 shows the evolution of the angular momentum of the merger remnant. We use Briggs figures (Briggs 1990) which are 2D polar coordinate representations of vector directions where the two spherical angle coordinates relative to a fixed reference frame, $\theta$ and $\phi$, are plotted as the radial and angle coordinates, respectively, on a $2 \mathrm{D}$ polar plot. The plot shows a Briggs figure for the stars from different GCs. It can be seen that the angular momentum vectors are well aligned to better than $10^{\circ}$ for stars inside of $4 R_{e}$. The one exception is GC2 after three GCs have merged, which is misaligned by $\sim 20^{\circ}$ from GC0 and GC1 probably due to its recent merger. We repeated this analysis for stars inside of $3 R_{e}$ and found a very similar alignment indicating that the contribution of the outer stars to the total angular momentum does not bias this result.

The top row in Fig. 7 plots the angular momentum perpendicular to the plane of overall rotation, $j_{z}$, versus energy for stars in three spherical radial ranges at $1.09 \mathrm{Gyr} . j_{z}$ is measured once the merger remnant has been centred and its angular momentum vector aligned with the $z$-axis, resulting in any flattening of the stars into a disc lying in the $x-y$ plane (c.f. Fig. 1). Stars in the inner radial bin have lower $j_{z}$, increasing outwards. There is no evidence of groupings of stars with distinct angular momentum signatures in these plots that would be indicative of separate populations. If we look at stars originating in different SCs, the only one that shows a significant difference in this plot is GC5, i.e. the last one to merge. The middle and bottom rows of Fig. 7 show a comparison of $j_{z}$ versus energy for GC0 and GC5 in the same three radial bins. GC5 shows significantly more stars with positive angular momentum in the inner two radial bins; however, there is still significant overlap in stellar distribution.

We also looked for substructure in simulation S1 by examining the orientation of stellar angular momentum vectors. There seems the greatest likelihood of finding substructure at $140 \mathrm{Myr}$ when mixing is less complete. Fig. 8 plots the mass-weighted distribution of angular momentum orientation in the $\theta, \phi$ plane for all stars in simulation $\mathrm{S} 1$ at $140 \mathrm{Myr}$ in three radial ranges. The inner stars within $4 R_{e}$ seem well mixed with a uniform distribution of stars over $\phi$ clustered around $\theta=0^{\circ}$. However, though this is also true in the range $4 R_{e}<r<6 R_{e}$, in addition two distinct small regions of high density of angular momentum vectors can be seen in this figure at $(\theta, \phi) \approx\left(20^{\circ},-115^{\circ}\right)$ and $\left(45^{\circ},-35^{\circ}\right)$. These represent stars that are not yet well mixed into the merger remnant and may 

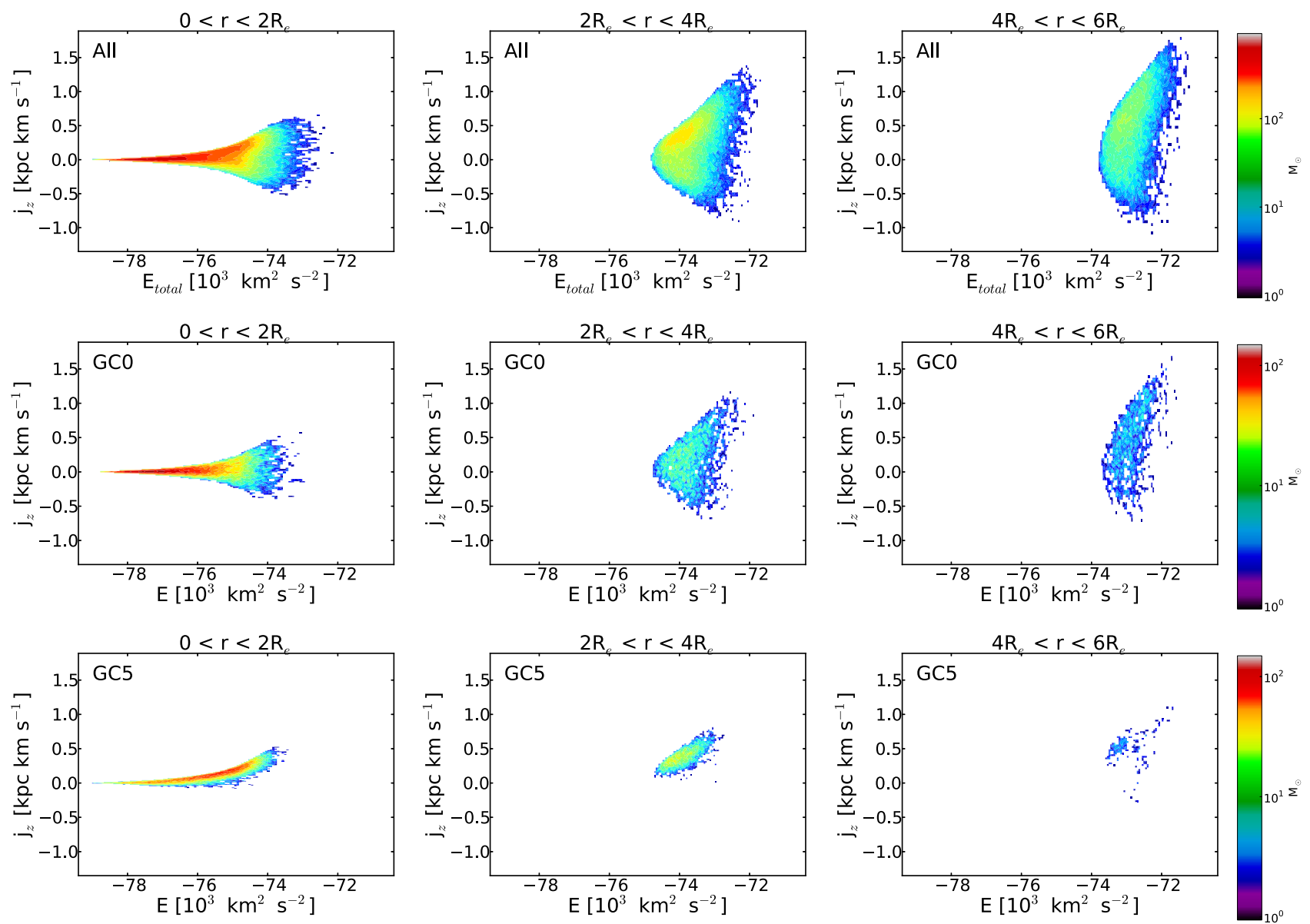

Figure 7. The vertical angular momentum $j_{z}$ versus energy for stars in three radial ranges at $1.09 \mathrm{Gyr}$ for simulation $\mathrm{S} 1$. The left-hand column shows stars between 0 and $2 R_{e}$, the middle between 2 and $4 R_{e}$, and the right-hand one shows stars between 4 and $6 R_{e}$. We show plots for all stars (top), for the stars originating in GC0 (middle), and GC5 (bottom). Stellar radii are measured in spherical coordinates. The mass of stars in each bin in the plot is colour coded with the maximum shown in dark red decreasing through yellow to blue.
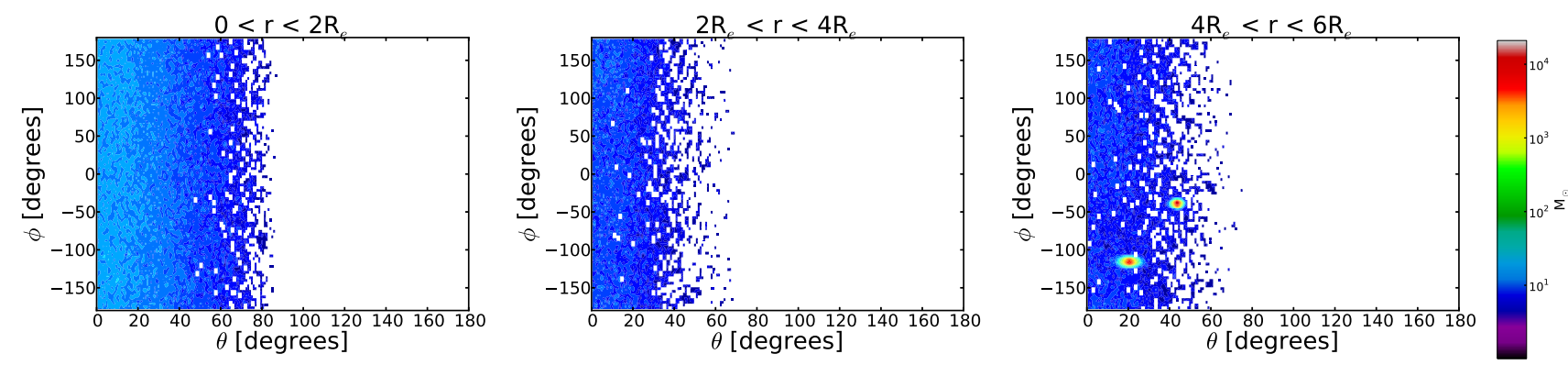

Figure 8. The orientation of angular momentum vectors for stars in three radial ranges after three clusters have merged (140 Myr) for simulation S1. The simulation is oriented so that the total angular momentum is oriented along the $z$-axis and the angular momentum spherical coordinates $\theta$ and $\phi$ are binned and a histogram of the mass is plotted. The left-hand column shows stars between 0 and $2 R_{e}$, the middle between 2 and $4 R_{e}$, and the right-hand one shows stars between 4 and $6 R_{e}$. Stellar radii are measured in spherical coordinates. The mass of stars in each bin in the plot is colour coded with the maximum shown in dark red decreasing through yellow to blue.

be distinguishable as stellar streams. This type of substructure is also seen following mergers at 280 and 1090 Myr in simulation S1.

Fig. 9 shows the evolution of the stars from each GC on the $(V / \sigma$, $\epsilon)$ diagram of Binney (2005). It has been measured as described in Hartmann et al. (2011) within $2 R_{e}$. The radial profiles of the observables depicted in the diagram have been calculated along a line of sight (LOS) corresponding to a direction perpendicular to the orientation of the angular momentum vector (i.e. edge-on). The region inside of $2 R_{e}$ is divided into bins of equal size and $V / \sigma$ is calculated as

$$
\left(\frac{V}{\sigma}\right)_{e} \equiv \frac{\left\langle V^{2}\right\rangle}{\left\langle\sigma^{2}\right\rangle}=\frac{\sum_{n=1}^{N} F_{n} V_{n}^{2}}{\sum_{n=1}^{N} F_{n} \sigma_{n}^{2}},
$$




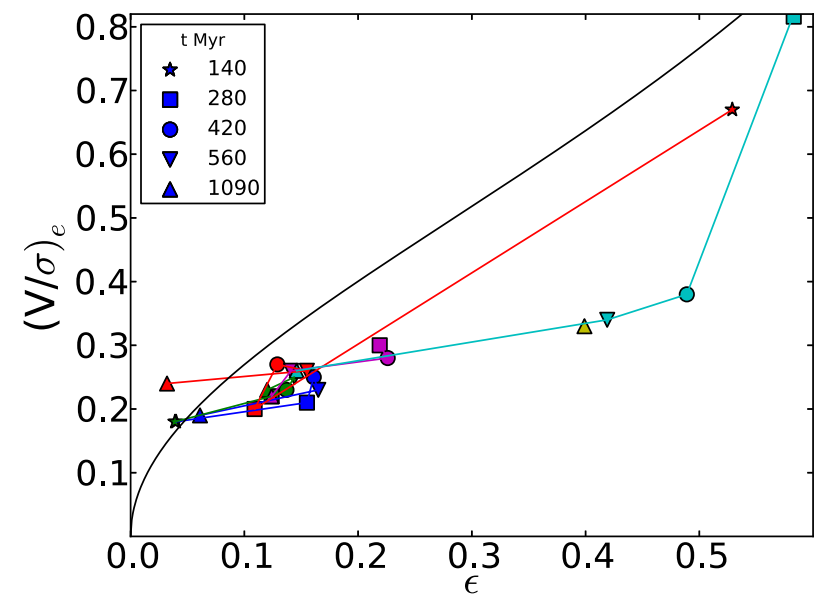

Figure 9. Evolution of the merger remnant of simulation $\mathrm{S} 1$ on the $(V / \sigma$, $\epsilon$ ) diagram of Binney (2005) measured as in Hartmann et al. (2011) within $2 R_{e}$. The black line shows the location of edge-on oblate isotropic rotator models. Each GC is indicated by a different colour. GC0 is blue, GC1 is green, GC2 is red, GC3 is magenta, GC4 is cyan, and GC5 is yellow. The different symbols show the values at different times. Note that GC3 and GC4 have not merged until $280 \mathrm{Myr}$ and have no data for $140 \mathrm{Myr}$, and GC5 has just merged at the end of the simulation and shows no evolution.

and the ellipticity $\epsilon$ is found from

$(1-\epsilon)^{2}=q^{2}=\frac{\left\langle y^{2}\right\rangle}{\left\langle x^{2}\right\rangle}=\frac{\sum_{n=1}^{N} F_{n} y_{n}^{2}}{\sum_{n=1}^{N} F_{n} x_{n}^{2}}$,

where $F_{n}$ is the mass in the $n$th bin and $V_{n}$ and $\sigma_{n}$ are the corresponding mean velocity and velocity dispersion in that bin. It can be seen that GCs when first merged can have large differences in their location on the diagram, for instance GC2, GC4, and GC5. However, as the remnant evolves, stars originating in different GCs move closer together. Even though GC2 and GC4 are initially located in very different parts of the diagram compared to the other mass components, they subsequently evolve towards the same region, corresponding to moderate flattening and mild rotation. In particular, the evolution in the diagram of GC2 (from a condition of high flattening and significant rotation) seems to be associated with merger events of GC3 and GC4. The component GC4 evolves in a similar way, but on a longer time-scale, and it becomes comparable to the other components only after the completion of all six merger events.

\subsubsection{Kolmogorov-Smirnov statistics}

In this section, we make some quantitative measurements of the probability that stars originating in each of the GCs could be distinguished from the overall distribution by using the KolmogorovSmirnov (K-S) test on the fractional distribution of stars within $4 R_{e}$. We shall assume that stars from each of the GCs can be chemically identified and then find the probability that stars from pairs of GCs can be distinguished. We analyse the spatial and kinematic properties of the stars in our simulations independently, reflecting the usual method used in observational studies cited in Section 1. This means that we only use either the stellar density or the kinematics separately to produce Figs 10 and 15 below.

We first perform $\mathrm{K}-\mathrm{S}$ tests on the spatial distribution of stars from each GC at the point where three, five, and six GCs make up the merger remnant. Fig. 10 shows a graphical representation of the probabilities, $p$ ( $p$-value), in $\log _{10}$ space, that stars originating in pairs of merged GCs are indistinguishable. The $p$-values are calculated on the cumulative fraction of a random selection of 200 stars from each cluster taken 1000 times and averaged. Fig. 10 is composed of $n^{2}$ cells where $n$ is the number of GCs merged in the remnant up to that point. The GCs are laid out along the $x$ and $y$ axes in order and the number displayed in the cell where the appropriate row and column cross is the $p$-value for the probability that the stars from those two GC populations are indistinguishable. The $p$-values in the upper left half of the figure (above the diagonal) are for the spatial distribution of the GCs and those in the lower right half (below the diagonal) are for the cumulative LOS velocity distribution. The LOS velocity is measured viewing the system perpendicular to the total angular momentum vector. The figure is colour coded so that high values of the $p$-value are darker (green) and low values are lighter (yellow).

The $p$-values for the LOS velocity show that there is a high probability that stars from any pair of GCs are indistinguishable in kinematics. The lowest $p$-value is $\sim 0.32$. The $p$-values for the spatial distribution show a greater likelihood that two GC populations could be distinguished. When the merger remnant consists of three GCs, GC0 and GC1 have a high probability that their stars are indistinguishable. However, the most recently merged GC2 has a relatively small probability that it is indistinguishable from GC0 or GC1, so it seems possible that it could be distinguished by this method. We see a similar situation when two more GCs have merged, at which point GC4 has less than 1 percent probability that its stars are indistinguishable from those of GC0 or GC1. However, all other $p$-values are $>1$ per cent implying that it would be difficult to distinguish separate populations. When the merger remnant contains six GCs even the most recently merged GC, GC5, has a greater than 2 per cent probability that its stars are spatially and kinematically indistinguishable from those of GC2. Other $p$-values are $>0.1$ apart from GC2 that remains the most distinguishable of the remaining GCs in its spatial distribution having a 4 per cent probability that its stars are indistinguishable from those of GC1.

\subsection{Properties of the merger remnant in simulation S2}

We now examine the results for simulation S2 (run A1 of Hartmann et al. 2011). In this simulation, a massive SC is initially placed on an orbit close to the centre where it eventually settles. A series of less massive SCs are then placed on orbits at $10 \mathrm{pc}$ from the centre, one at a time. Their orbits decay to the centre where they merge with the central structure. Each one is allowed to merge before the next one is added to the simulation. At the end of the simulation, 27 GCs have merged. Fig. 11 shows a stellar density map for simulation $\mathrm{S} 2$ at the end of the simulation. The merger remnant is much more flattened than that in simulation S1 because the GC orbits are all co-planar (compare with Fig. 2). Table 3 shows the mass and halfmass radius for the merger remnant at 115, 220, and $300 \mathrm{Myr}$ when 10,20 , and 27 clusters have merged. $R_{e}$ grows significantly between 115 and $220 \mathrm{Myr}$ from 2.9 to $6.2 \mathrm{pc}$.

Fig. 12 shows the evolution of the surface-density profile for the merger remnant within $4 R_{e}$ showing increasing density at the centre. Fig. 13 shows surface-density profiles for specific GCs at the same three times. The GCs which merge first have very similar density profiles but we see larger differences for the most recently merged GCs, especially at later times. The density profile for these GCs is either flat in the middle or even dropping towards the centre, showing that their density profiles will evolve further. 

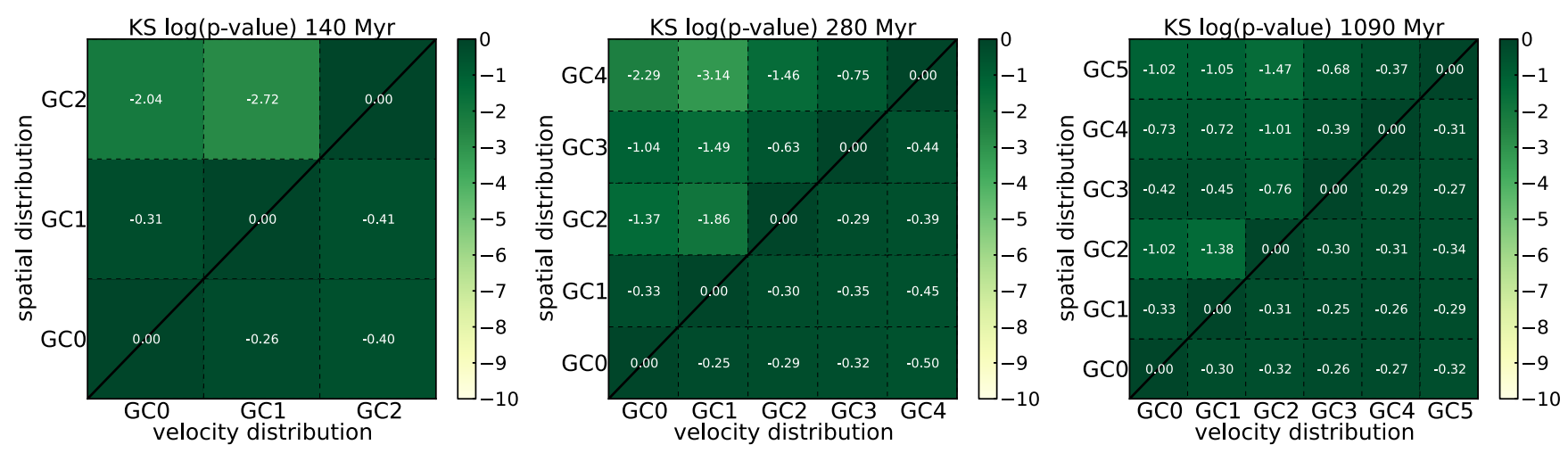

Figure 10. Simulation $\mathrm{S} 1 \log _{10}$ of $p$-values for 200 stars from each cluster within $4 R_{e}$ when the merger remnant contains three GCs (left), five GCs (middle), and six GCs (right). The grid is arranged so that the figures above the diagonal are calculated by comparing the cumulative spatial distributions of a pair of GCs and those below the diagonal are based on a comparison of the cumulative absolute velocity distribution as seen by an observer viewing the system perpendicular to the total angular momentum vector. The $p$-value for any pair of GCs is found at the intersection of the appropriate row and column. The figure is colour coded so that high $p$-values are darker (green) and low $p$-values are lighter (yellow).
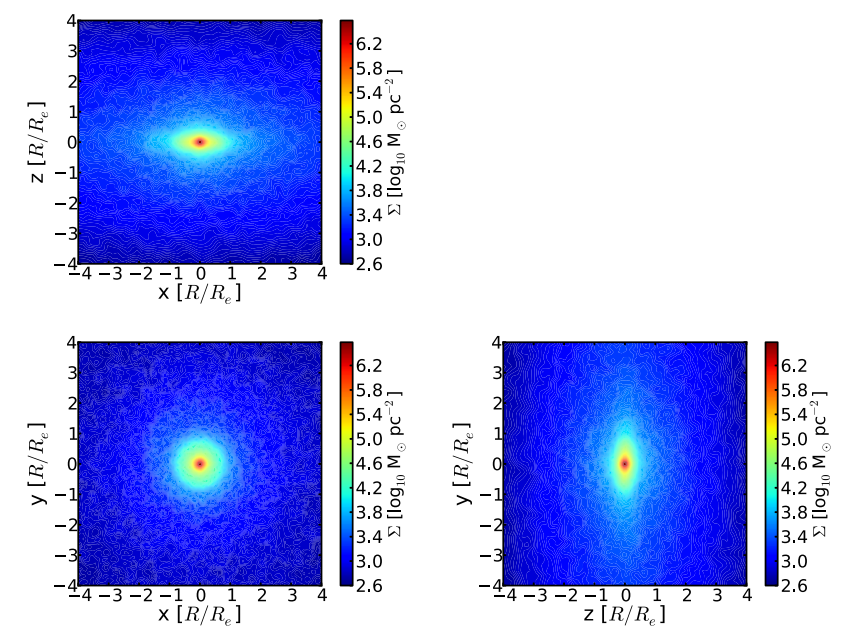

Figure 11. Surface-density map of the merger remnant from simulation S2 (run A1 of Hartmann et al. 2011) showing three orthogonal projections. The lower left-hand plot is face-on to the net angular momentum vector and the other two are perpendicular to it.

Fig. 14 depicts the velocity dispersion profiles for the same GCs at the three times shown in Figs 11 and 12 as well as the velocity dispersion for the whole cluster. These profiles show a similar pattern to the density and kinematic profiles. The first five GCs to merge have very similar profiles and the most recent GC to have merged always shows the biggest difference. There are also bigger differences for the recently merged GCs at later times. The velocity dispersions for the whole cluster increase significantly from when the merger remnant contains 10 GCs to when it has 27 GCs.

At each time the five most recently merged GCs all show a greater difference in density profile and kinematics from the average. From inspection of the density profiles, it appears that these components are still experiencing significant evolution, and therefore it is not surprising to notice some differences in their kinematics. After the first 10 merger events, only the last cluster component (GC9) is still distinguishable from the global behaviour. Similarly, after 20 merger events, the behaviour of the last five clusters still retains some differences from the overall spatial and kinematic profiles

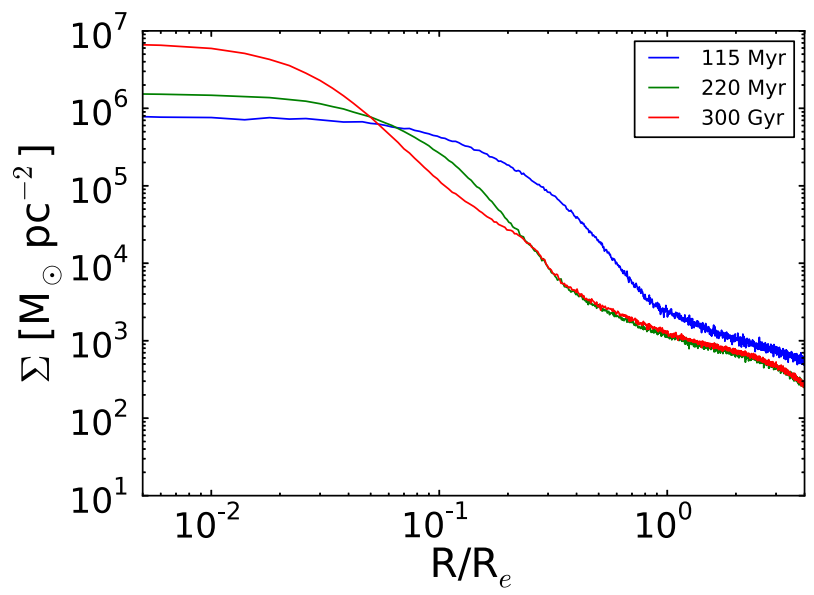

Figure 12. Evolution of the surface density for the merger remnant in simulation $\mathrm{S} 2$.

(especially in the case of GC19). This applies also to the subsequent components in the remnant, after it has experienced the full series of 27 merger events. It appears that in simulation S2, there is a greater likelihood that we would be able to distinguish individual populations from specific recently merged GCs but later mergers produce a smaller fractional perturbation.

\subsubsection{Kolmogorov-Smirnov statistics}

We performed $\mathrm{K}-\mathrm{S}$ tests for simulation S2, looking at the spatial distribution of stars originating in individual merged GCs after 10, 20, and 27 GCs have merged, drawing 200 stars from each cluster in each sample. Fig. 15 shows a representative sample of cumulative fractions of stars originating in individual merged GCs at the same times considered in Fig. 15. The pairs are defined by considering a selection of components that belong to either one of the five earliest merged GCs or from one of the five most recently merged clusters; such a prescription allowed us to perform a comparison between the most similar and the most different distributions, respectively. The pair from the five earliest merged GCs were chosen to have the greatest apparent difference in distribution. After $20 \mathrm{GCs}$ and 27 GCs have merged a further GC was chosen from an intermediate 

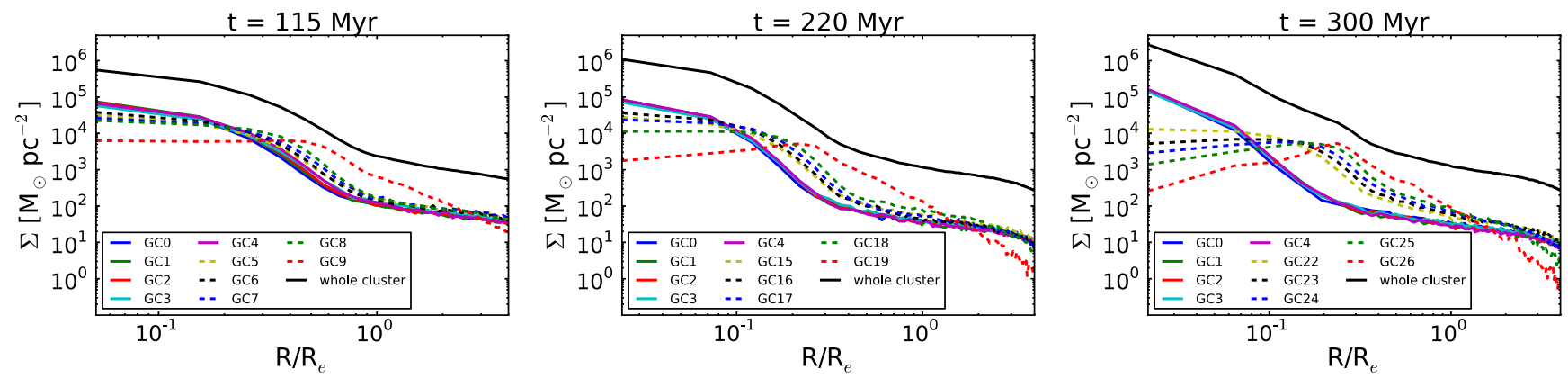

Figure 13. The density profile at three different times for model 2. The panels show when $10 \mathrm{GCs}$ (left), $20 \mathrm{GCs}$ (centre), and $27 \mathrm{GCs}$ (right) are in the merger remnant.
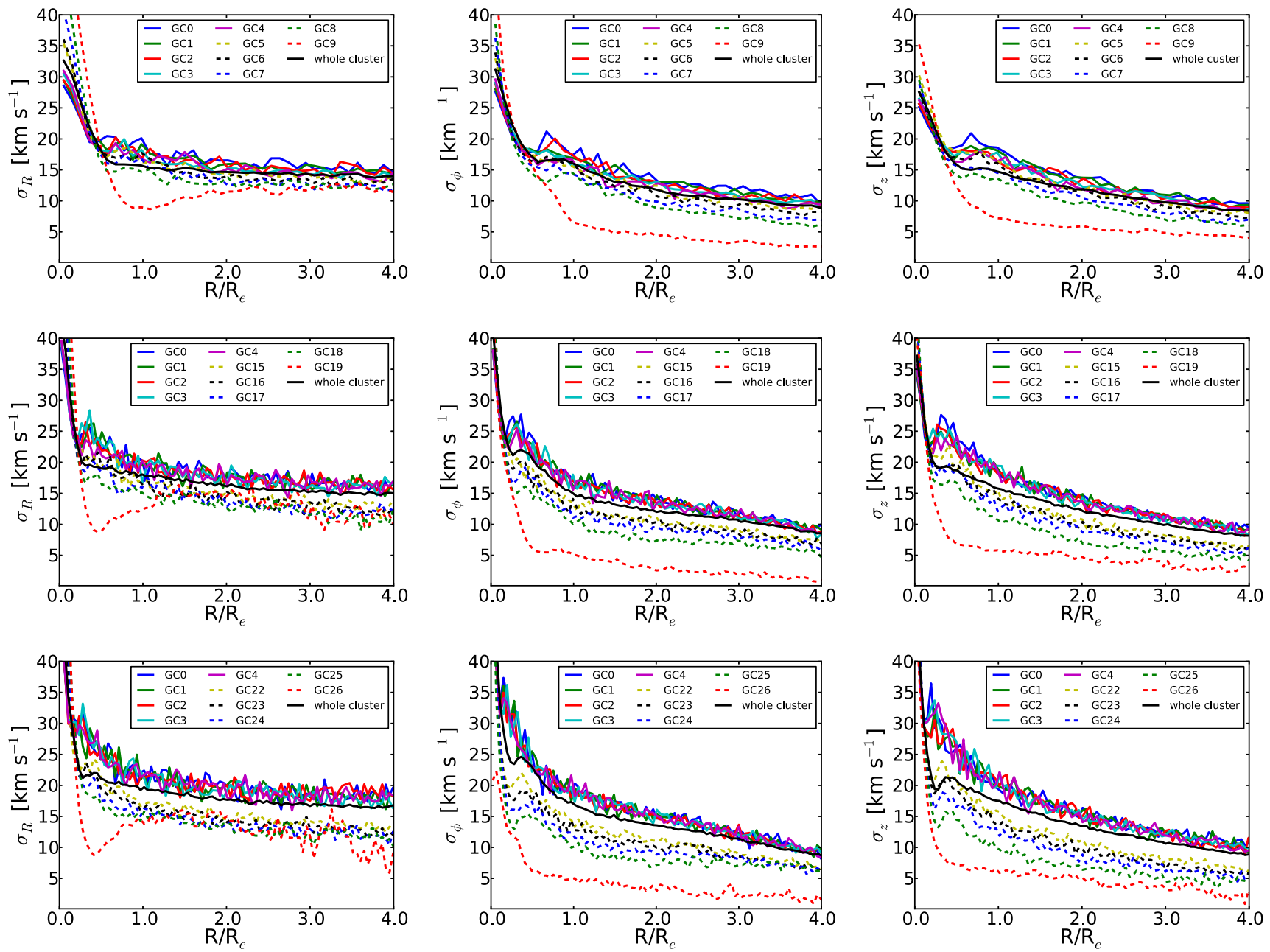

Figure 14. Simulation S2 velocity dispersion profiles in cylindrical coordinates when the merger remnant contains 10 GCs (top), 20 GCs (middle), and 27 GCs (bottom). Profiles for the first five GCs to merge and the five most recently merged GCs are shown. Radial profiles are on the left, azimuthal profiles are in the centre, and vertical profiles are on the right.

merger event to sample a different stage of the merger. The objective of this selection is to show the outliers of the possible comparisons in spatial segregation.

Again the $p$-values in the upper left half of the figure (above the diagonal) are for the spatial distribution of the GCs and those in the lower right half (below the diagonal) are for the LOS velocity seen by an observer viewing the system perpendicular to the total angular momentum vector. The $p$-values for the LOS velocity tests again show that there is a high probability that any pair of $\mathrm{GCs}$ are indistinguishable using this $\mathrm{K}-\mathrm{S}$ test. The lowest $p$-value, $\sim 0.14$, still demonstrates a high likelihood that the two GCs are indistinguishable. When the merger remnant contains $10 \mathrm{GCs}$ the $\mathrm{K}-\mathrm{S}$ tests based on the spatial distribution show that the earliest merged GCs, which are GC0, GC4, and GC5, taken pairwise have $p$-values $>1$ per cent, whereas GC9 has a low probability that it is indistinguishable from the other three. Similarly, when 20 GCs have 

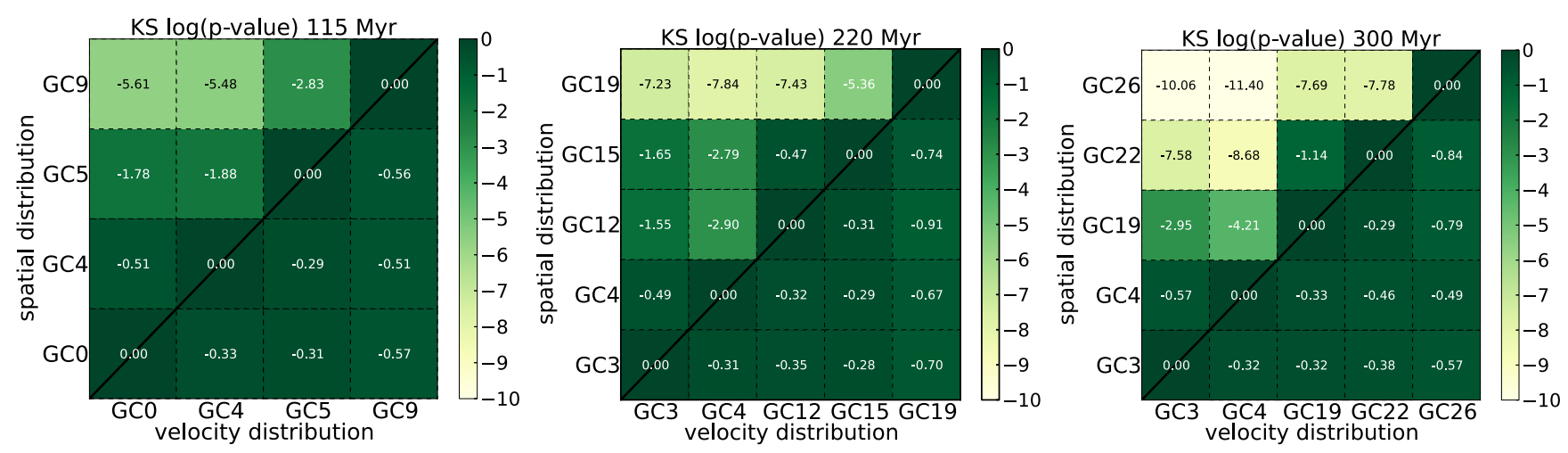

Figure 15. Simulation $\mathrm{S} 2 \log _{10}$ of $p$-values for the cumulative spatial distribution of 200 stars from each cluster within $4 R_{e}$ when the merger remnant contains $10 \mathrm{GCs}$ (left), $20 \mathrm{GCs}$ (middle), and $27 \mathrm{GCs}$ (right), and cumulative absolute velocity distribution as seen by an observer viewing the system perpendicular to the total angular momentum vector. The $p$-value for any pair of GCs is found at the intersection of the appropriate row and column. The figure is colour coded so that high $p$-values are green and low $p$-values are yellow. See Fig. 10 for a full explanation of the layout.

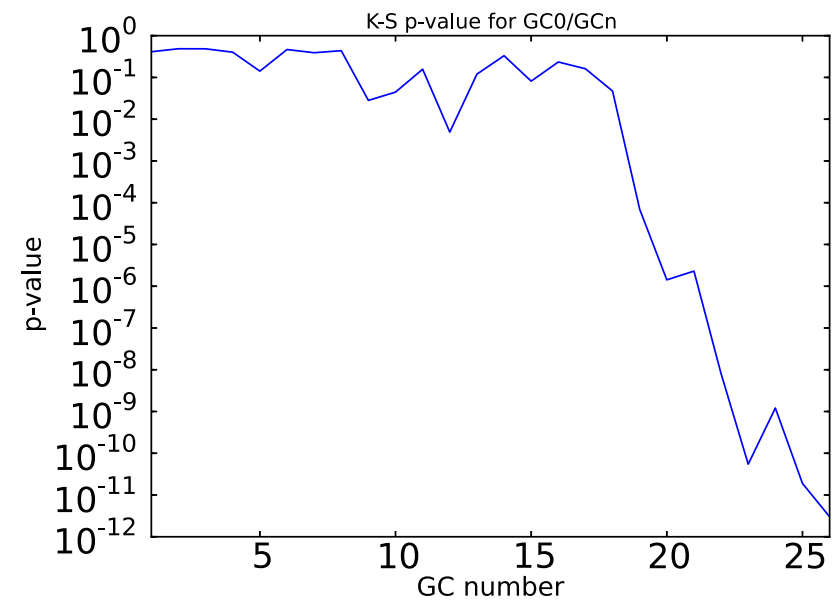

Figure 16. The probability when the merger remnant is made of $27 \mathrm{GCs}$ that the earliest merged GC0 in simulation S2 is indistinguishable from all the others based on spatial distribution with samples of 200 stars from each GC.

merged GC3, GC4, GC12, and GC15, which have been merged for longest, have $p$-values $>0.001$ in tests between each other, whereas GC19 has a $p$-value $<5 \times 10^{-6}$ when tested pairwise with any of the others. When 27 GCs have merged, GC3 and GC4 have a similar spatial distribution and GC19, GC22, and GC26 have low likelihoods of being indistinguishable from GC3 and GC4.

At each time, we find that the 6-8 most recently merged GCs have a low probability that they are indistinguishable from earlier merged GCs based on their spatial distribution. When GCs have merged prior to this in the merger sequence, their stars have a spatial distribution that is similar to that of the overall merger remnant. An illustration of this is shown in Fig. 16. This shows the probability, based only on spatial distributions, that the earliest merged GC, GC0, is indistinguishable from each of the others when the merger remnant contains stars from $27 \mathrm{GCs}$. This $p$-value is generally $\sim 0.2-0.5$ but always $>1$ per cent for all GCs up to GC18 and then falls sharply from GC19 (probability, $p<10^{-5}$ ) to GC26 (just merged). This implies that stars from a GC that has merged in the most recent eight mergers could be distinguished by their spatial distribution but stars from a GC that underwent a prior merger could not.

Fig. 17 shows the $p$-values laid out as before for all pairs of GCs at the end of the simulation in simulation S2. The lower right half of the figure shows the $p$-values for the velocity distributions. These $p$-values are all $>10$ per cent showing we cannot use their velocity distributions to distinguish stars from different GCs. Based on these results, it seems more likely for observations to find substructure due to variations in stellar density than in stellar kinematics. The upper left half of the figure shows $p$-values for the spatial distributions. The $p$-values of the last six to eight GCs to merge when tested with one of the first 18 GCs to merge are low. $p$-values for GC0-GC18 taken in pairs show higher likelihood that these GCs are indistinguishable.

If we look for groups of GCs that all have $p$-values of $>10$ per cent when tested with each other, we find two large groups made up of 11 and 7 GCs and three small groups of three, three, and two GCs. The two large groups are made up from the first 18 GCs to merge. If we set the $p$-value threshold at 1 per cent, we still find two large groupings made up of 15 and 6 GCs (again made up from the earlier GCs to merge) and two smaller made up of three and two GCs.

\subsection{Dependence of $\mathrm{K}-\mathrm{S}$ results on number of stars observed}

We have chosen, somewhat arbitrarily, 200 stars from each cluster to compare our distributions. Given that we have simulations with $\sim 40000$ star particles representing each GC, we could have used up to thousands of stars to perform our $\mathrm{K}-\mathrm{S}$ tests. Though the profiles we have examined would not have changed by using more points, this would result in lower $p$ values for the same difference in spatial or velocity distribution. Observational uses of the $\mathrm{K}-\mathrm{S}$ test are limited by the number of stars observed and so for our purposes we should ensure that we are performing our tests with a similar number of stars. Kučinskas et al. (2014) perform their K-S tests on 101 main-sequence turn-off stars in 47 Tuc meaning they are comparing subsamples with tens of stars. Lardo et al. (2011) used $\mathrm{K}-\mathrm{S}$ tests to distinguish different populations of stars in the $u, g, r$ SDSS bands. They compared samples containing from tens to several hundred stars. In order to assess the effect of using larger numbers of stars, we repeated some of our K-S tests again with 1000 stars from each cluster.

Fig. 18 shows the $p$-values as described in Section 3.1.3 but now with 1000 stars from each cluster. At $140 \mathrm{Myr}$, the results are similar to the results with 200 stars per cluster. As previously, GC2 is distinguishable by its spatial distribution. At $280 \mathrm{Myr}$, however, most GC pairs have a spatial $p$-value $<10^{-6}$ and are now distinguishable by their spatial distribution. Only GC0/GC1 and GC2/GC3 have a $p$-value for their spatial distribution $>1.5$ per cent. We see a similar 


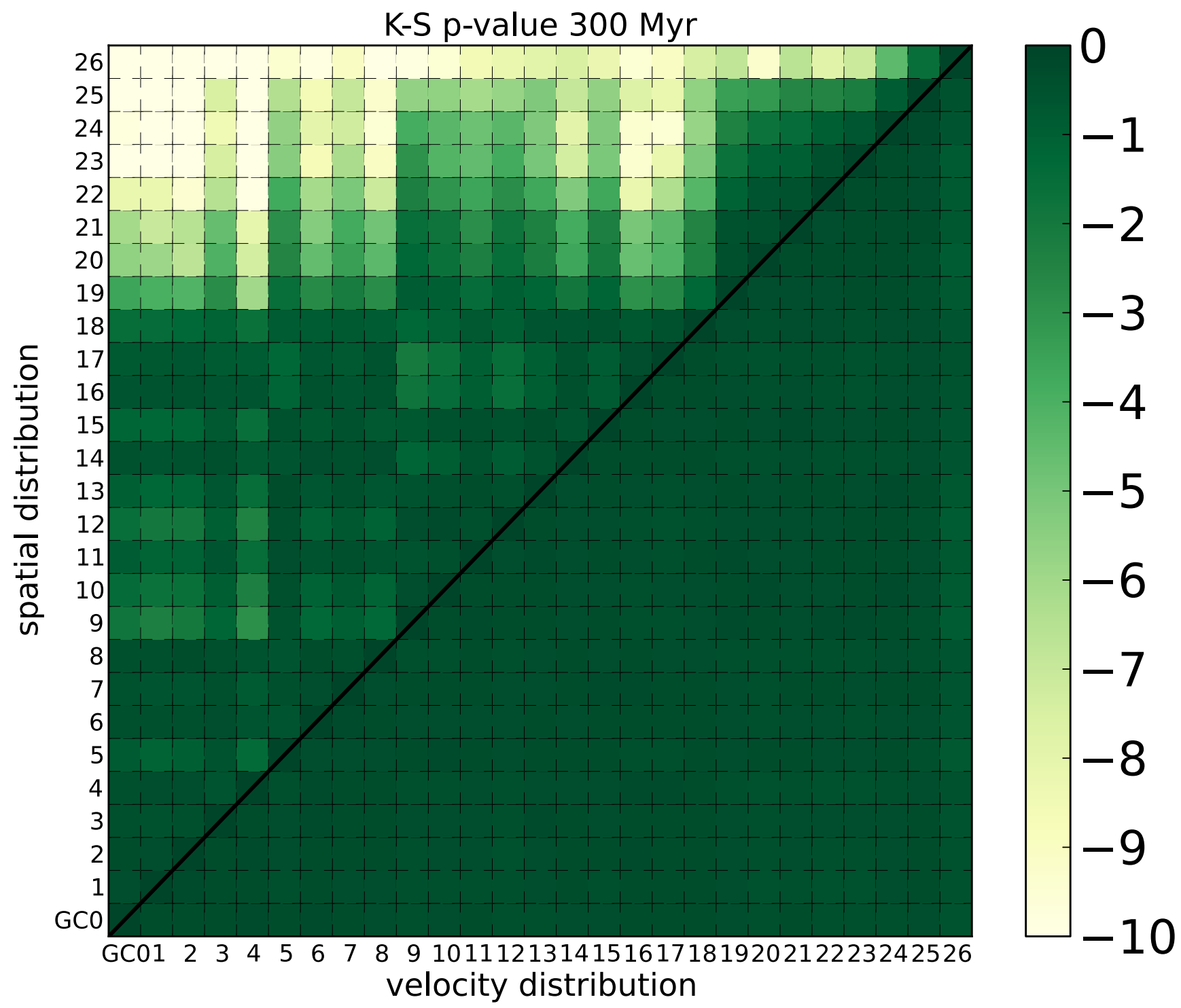

Figure 17. $p$-values for the cumulative spatial and velocity distribution of 200 stars within $4 R_{e}$ at the end of the simulation for simulation S2, for all pairs of GCs. The $p$-value for any pair of GCs is found at the intersection of the appropriate row and column. The figure is colour coded so that high $p$-values are green and low $p$-values are yellow. See Fig. 10 for a full explanation of the layout.

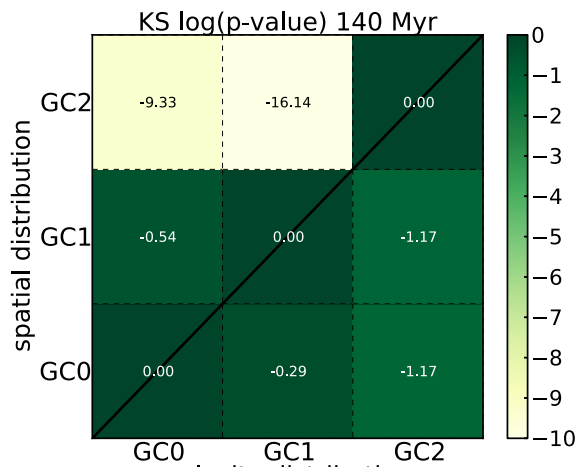

velocity distribution
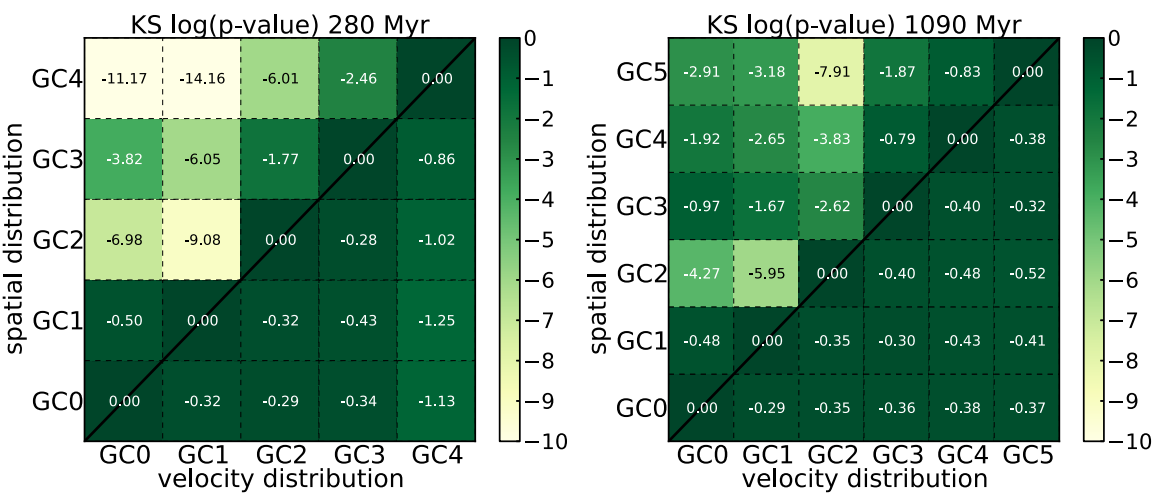

Figure 18. Simulation $\mathrm{S} 1 \log _{10}$ of $p$-values for the cumulative spatial distribution of 1000 stars from each cluster within $4 R_{e}$ when the merger remnant contains three GCs (left), five GCs (middle), and six GCs (right) and cumulative absolute velocity distribution as seen by an observer viewing the system perpendicular to the total angular momentum vector as in Fig. 10. The $p$-value for any pair of GCs is found at the intersection of the appropriate row and column. The figure is colour coded so that high $p$-values are green and low $p$-values are yellow. See Fig. 10 for a full explanation of the layout. 
effect at $1090 \mathrm{Myr}$ though not quite as marked. However, at this time GC2, GC4, and GC5 are distinguishable by their spatial distribution with GC3, GC4, and GC5 having relatively high $p$-values that they are drawn from the same population. Thus, unsurprisingly, increasing the number of stars has had a significant effect on our ability to identify different populations by their spatial distribution. Pairs that were previously indistinguishable can now be distinguished from their spatial distributions.

\section{DISCUSSION}

We have shown that the stars originating in individual GCs that merge can be difficult to identify from their spatial or velocity distributions with currently observable sample sizes. It is often the most recently merged GCs that are distinguishable by observations. Stars from the most recently merged few GCs have a low probability, based on their spatial distribution, so that they are indistinguishable from earlier merged GCs. However, this probability increases quickly as more GCs merge and soon become spatially distributed and kinematically similar to the rest of the cluster. Our analysis has treated the spatial and kinematic stellar distributions of our simulations independently, reflecting the current observational approach. It is likely that examining the combined spatial and kinematic properties by looking for correlations in phase space would make it possible to detect substructure for longer.

Our simulations show examples of violent relaxation (LyndenBell 1967) where the final distribution of particles is the result of the star particles being scattered by the rapidly changing gravitational potential produced by a merger. This is true in simulation $\mathrm{S} 1$ where the accreting GC will produce changes in the gravitational potential comparable to the existing merger remnant gravitational field. In simulation S2, the accreting GCs have a smaller mass ratio with the existing SC but still produce substantial perturbations in the merger remnant potential. Studies of violent relaxation in galaxy mergers have found that radial abundance and colour gradients can survive the mixing of stellar populations but that they are reduced (White 1980; Barnes 1988, 1996; Mihos \& Hernquist 1994).

Our merger remnants are collisionless, non-spherical systems. Merritt \& Valluri (1996) found that collisionless mixing in triaxial potentials representative of elliptical galaxies occurs with characteristic times of 10-30 dynamical times. For our systems, the dynamical time at $R_{e} \sim 5 \times 10^{5} \mathrm{yr}$ giving a mixing time of 5 to $15 \mathrm{Myr}$. Valluri et al. (2007) studied the mechanisms responsible for mixing in collisionless mergers (in their case dark matter haloes). They found that the mixing in phase space is driven by the exchange of energy and angular momentum at pericentric passage due to tidal shocks and dynamical friction. They find that in the merger remnant most particles retain a memory of their original kinetic energy and angular momentum but there are changes due to the tidal shocks. Importantly, they do not find more large-scale mixing in radius compared to an isolated halo and conclude that radial gradients in stellar properties, such as metallicity, can survive such mergers. In the case of NSCs (and potentially GCs too), we would like to know if the merging of stellar systems each composed of a single population can produce multiple populations distinguishable by their spatial and velocity profiles. If mono-abundance GCs merge to form NSCs, they will retain some of their kinetic energy and angular momentum profiles. Stars from different merging GCs will have similar spatial and velocity distributions prior to the merger and retain these afterwards. Our results indicate that creating a merger remnant results in the stars from different GCs having a similar spatial and velocity distribution except for recent mergers, implying that if our clusters were made up of distinct stellar populations they would be difficult to detect by their spatial and velocity distribution.

In contrast, observations of the most massive GCs in the Galaxy show that different populations can be identified by their chemistry or photometry and that these populations have distinguishable spatial and velocity distributions. Kučinskas et al. (2014) studied 47 Tuc and found a difference in $[\mathrm{Na} / \mathrm{O}]$ and $[\mathrm{Li} / \mathrm{Na}]$ abundance ratios. They identified three distinct groupings and found that these groups were distinguishable in both their spatial distribution and kinematic properties. Richer et al. (2013) also found that populations identified by colour in 47 Tuc could be distinguished by their radial distribution as well as their proper motions. Lardo et al. (2011) in a study of nine Galactic GCs found that populations could be identified by colour differences and these populations could be distinguished by their radial distribution.

Kobayashi (2004) studied the chemodynamic evolution of elliptical galaxies following mergers and showed that metallicity gradients have the largest change when the galaxies are of comparable mass. They find that when the mass ratio of the two galaxies is more than 20 per cent, then the metallicity gradient change is $\gtrsim 0.5$ dex. Di Matteo et al. (2009) investigated dry mergers of early-type galaxies with a variety of properties using $N$-body simulations. They found that such mergers flatten the metallicity gradient in the merger remnant but that ellipticals can retain their pre-merger metallicity gradient if one of the merging galaxies has a steep pre-merger slope. Should a small metallicity gradient exist in our merger remnant, the repeated merging in our simulations would be likely to continually reduce any stellar population gradients making any remnant gradient hard to observe. Building an NSC from GCs would require early mergers to have mass ratios of more than 20 per cent maximizing the reduction in the existing gradient. This is the case in our earliest mergers in simulation S1 where we find the greatest likelihood of distinguishing stars from different merging GCs.

From the GCs perspective, the characterization of the process of mixing of different stellar populations plays a crucial role for the interpretation of the spatial and kinematical properties of present-day Galactic SCs. The key physical driver of the mixing is represented by two-body collisional relaxation processes, which, during the course of the long-term dynamical evolution of the systems, may gradually erase any intrinsic difference in the spatial and kinematical distribution of different stellar populations. Within the formation scenario in which the AGB stars are the 'polluters' contributing to enrich the gas from which the second generation is formed, Vesperini et al. (2013) have explored, by means of direct $N$-body simulations, the time-scales and the dynamics of the spatial mixing of two different populations, and their dependence on the initial concentration of the 'second generation' stars. They found that the time-scale for complete mixing depends on the initial concentration of the second generation, but that, in general, complete mixing is expected only for clusters in the late stages of their evolution, after they have lost a significant fraction of their initial mass due to relaxation-driven processes. Such a theoretical investigation therefore supports the observational evidence that, in several present-day SCs, different populations are characterized by distinguishable spatial distributions (with the helium-enriched population being the more centrally concentrated one). In particular, Kučinskas et al. (2014), in a study of 47 Tuc, found that a K-S test of the fractional distribution of the different generations of stars plotted against radius from the centre of the cluster gives a probability $p=6.0 \times 10^{-7}$ that the primordial and chemically enriched distributions are indistinguishable (for a total of 101 stars). K-S tests of the absolute LOS velocities of the different stellar generations also give low probabilities that they are 
drawn from the same population $\left(p=7.0 \times 10^{-7}\right)$. We emphasize that $\mathrm{K}-\mathrm{S}$ tests with a greater number of stars from mass components associated with specific GCs in our simulations find much lower likelihoods that they are drawn from the same population both for spatial and velocity distributions.

It should be noted that the initial density profile of our GCs would have an effect on the degree of mixing in our merger remnants. Assmann et al. (2013) noted that initially flat inner density profiles favour preservation of pre-existing structures compared to steeper cuspy ones. Our initial density profile is cuspy, with the density of the bulge model scaling as $r^{-1}$ inside $100 \mathrm{pc}$. This would lead to a lower likelihood of preserving observable populations; however, the overall density profile is flattened by the earliest mergers inside a few $R_{e}$ in both our simulations, favouring the preservation of structure. Gavagnin, Mapelli \& Lake (2016) found that the key aspects of the initial conditions for mergers of GCs are the initial mass and density ratios of the progenitors. They found that more massive progenitor GCs dominate except where the less massive progenitor is denser by a similar factor to the mass ratio. The mass ratios in our mergers are a few to one for simulation S1 indicating that this would not have a significant effect on the results. The ratios are larger for simulation $\mathrm{S} 2$, but as low as 3:1 for the early mergers and less than 20:1 for the majority of the mergers.

As for the GCs kinematic properties, it has been shown, via direct $N$-body simulations, that different populations may be characterized by different kinematical properties (see section 4 in Bellini et al. 2015). In particular, the diffusion from the innermost regions to the outer parts of the clusters of the most centrally concentrated population is associated with the growth of radial anisotropy in such a population, in agreement with recent observational studies of selected Galactic GCs (47 Tuc, Richer et al. 2013; NGC 2808, Bellini et al. 2015). One additional question is related to the kinematic imprints (and their survival) of different formation scenarios for multiple stellar populations in GCs. Hénault-Brunet et al. (2015) have addressed such a question by using direct $N$-body simulations, and found that different formation mechanisms show distinct kinematical signatures that can persist for a Hubble time. In summary, in the context of the formation and dynamical evolution of GCs, there is convincing evidence, mostly based on $N$-body models, that spatial and kinematical differences, either intrinsically associated with the formation scenarios or induced by collisional relaxation processes, may persist for several half-mass relaxation times.

Bearing in mind the fundamental differences between the formation scenarios (and the intrinsic nature) of GCs and NSCs, we have performed an investigation of the structural and kinematical properties of the mass components associated with different clusters, progressively merged to form a single central stellar system. Motivated by specific cases of peculiar SCs that, in light of their chemical and dynamical complexity, have been suggested to be stripped nuclei of dwarf galaxies (e.g. M 54, $\omega$ Cen), we assess the existence and persistence of any spatial or dynamical signature associated with the merger histories considered in our two simulations. Our analysis shows that such a differentiation is difficult with currently available numbers of observations if NSCs formed by merging alone, except for recent mergers, and hints towards the need for gas and dissipational processes in observed systems.

\section{ACKNOWLEDGEMENTS}

We would like to thank Iskren Georgiev, Nadine Neumayer, and Alessandra Mastrobuono-Battisti for their useful discussions and comments on the earlier drafts of this paper. We would also like to thank the referee Mark Wilkinson for his very helpful comments and questions. Simulations in this paper were carried out on the COSMOS Shared Memory system at DAMTP, University of Cambridge operated on behalf of the STFC DiRAC HPC Facility. This equipment is funded by BIS National E-infrastructure capital grant ST/J005673/1 and STFC grants ST/H008586/1 and ST/K00333X/1. DRC and VPD were supported by STFC Consolidated grant no. ST/J001341/1. We made use of PYNBODY (https://github.com/pynbody/pynbody) in our analysis for this paper. A-LV acknowledges support from the Royal Commission for the Exhibition of 1851. We would like to acknowledge the Lorentz Centre in Leiden that hosted the meeting 'Nuclear Star Clusters in Galaxies, and the Role of the Environment', 2014 June 30 to July 4, where discussions stimulated our ideas set forth in this paper.

\section{REFERENCES}

Amaro-Seoane P., Konstantinidis S., Brem P., Catelan M., 2013, MNRAS, 435,809

Anderson J., van der Marel R. P., 2010, ApJ, 710, 1032

Antonini F., 2013, ApJ, 763, 62

Antonini F., 2014, ApJ, 794, 106

Antonini F., Capuzzo-Dolcetta R., Mastrobuono-Battisti A., Merritt D., 2012, ApJ, 750, 111

Antonini F., Barausse E., Silk J., 2015, ApJ, 812, 72

Arca-Sedda M., Capuzzo-Dolcetta R., 2014, MNRAS, 444, 3738

Arca-Sedda M., Capuzzo-Dolcetta R., Antonini F., Seth A., 2015, ApJ, 806, 220

Assmann P., Fellhauer M., Wilkinson M. I., Smith R., Blaña M., 2013, MNRAS, 435, 2391

Barnes J. E., 1988, ApJ, 331, 699

Barnes J., 1996, in Bender R., Davies R. L., eds, Proc. IAU Symp. 171, New Light on Galaxy Evolution. Kluwer, Dordrecht, p. 191

Bastian N., 2015, preprint (arXiv:1510.01330)

Bastian N., Lamers H. J. G. L. M., de Mink S. E., Longmore S. N., Goodwin S. P., Gieles M., 2013, MNRAS, 436, 2398

Bekki K., 2007, PASA, 24, 77

Bekki K., Norris J. E., 2006, ApJ, 637, L109

Bekki K., Yong D., 2012, MNRAS, 419, 2063

Bellini A. et al., 2015, ApJ, 810, L13

Bianchini P., Varri A. L., Bertin G., Zocchi A., 2013, ApJ, 772, 67

Binney J., 2005, MNRAS, 363, 937

Böker T., Laine S., van der Marel R. P., Sarzi M., Rix H.-W., Ho L. C., Shields J. C., 2002, AJ, 123, 1389

Briggs F. H., 1990, ApJ, 352, 15

Capuzzo-Dolcetta R., 1993, ApJ, 415, 616

Capuzzo-Dolcetta R., Miocchi P., 2008a, MNRAS, 388, L69

Capuzzo-Dolcetta R., Miocchi P., 2008b, ApJ, 681, 1136

Carollo C. M., Stiavelli M., de Zeeuw P. T., Mack J., 1997, AJ, 114, 2366

Carretta E., 2015, ApJ, 810, 148

Carretta E. et al., 2010a, A\&A, 520, A95

Carretta E. et al., 2010b, ApJ, 714, L7

Carretta E., Lucatello S., Gratton R. G., Bragaglia A., D’Orazi V., 2011, A\&A, 533, A69

Carson D. J., Barth A. J., Seth A. C., den Brok M., Cappellari M., Greene J. E., Ho L. C., Neumayer N., 2015, AJ, 149, 170

Catelan M., 1997, ApJ, 478, L99

Cen R., 2001, ApJ, 560, 592

Cordero M. J., Pilachowski C. A., Johnson C. I., McDonald I., Zijlstra A. A., Simmerer J., 2014, ApJ, 780, 94

Côté P. et al., 2006, ApJS, 165, 57

D’Antona F., Vesperini E., D'Ercole A., Ventura P., Milone A. P., Marino A. F., Tailo M., 2016, MNRAS, 458, 2122

D’Ercole A., Vesperini E., D’Antona F., McMillan S. L. W., Recchi S., 2008, MNRAS, 391, 825 
D’Ercole A., D'Antona F., Ventura P., Vesperini E., McMillan S. L. W., 2010, MNRAS, 407, 854

D'Ercole A., D’Antona F., Carini R., Vesperini E., Ventura P., 2012, MNRAS, 423, 1521

D’Souza R., Rix H.-W., 2013, MNRAS, 429, 1887

Da Costa G. S., Held E. V., Saviane I., Gullieuszik M., 2009, ApJ, 705, 1481

De Lorenzi F., Hartmann M., Debattista V. P., Seth A. C., Gerhard O., 2013, MNRAS, 429, 2974

de Mink S. E., Pols O. R., Langer N., Izzard R. G., 2009, A\&A, 507, L1

Debattista V. P., Sellwood J. A., 2000, ApJ, 543, 704

Di Matteo P., Pipino A., Lehnert M. D., Combes F., Semelin B., 2009, A\&A, 499, 427

Emsellem E., van de Ven G., 2008, ApJ, 674, 653

Ferraro F. R. et al., 2009, Nature, 462, 483

Figer D. F., McLean I. S., Morris M., 1999, ApJ, 514, 202

Figer D. F. et al., 2002, ApJ, 581, 258

Gavagnin E., Mapelli M., Lake G., 2016, MNRAS, 461, 1276

Georgiev I. Y., Böker T., 2014, MNRAS, 441, 3570

Gnedin O. Y., Ostriker J. P., 1997, ApJ, 474, 223

Gnedin O. Y., Ostriker J. P., Tremaine S., 2014, ApJ, 785, 71

Gratton R. G., Carretta E., Bragaglia A., 2012, A\&AR, 20, 50

Harris W. E., 1996, AJ, 112, 1487

Hartmann M., Debattista V. P., Seth A., Cappellari M., Quinn T. R., 2011, MNRAS, 418, 2697

Hénault-Brunet V., Gieles M., Agertz O., Read J. I., 2015, MNRAS, 450, 1164

Hernquist L., 1990, ApJ, 356, 359

Ibata R. et al., 2009, ApJ, 699, L169

Kobayashi C., 2004, MNRAS, 347, 740

Kučinskas A., Dobrovolskas V., Bonifacio P., 2014, A\&A, 568, L4

Lanzoni B. et al., 2010, ApJ, 717, 653

Lapenna E., Mucciarelli A., Lanzoni B., Ferraro F. R., Dalessandro E., Origlia L., Massari D., 2014, ApJ, 797, 124

Lardo C., Bellazzini M., Pancino E., Carretta E., Bragaglia A., Dalessandro E., 2011, A\&A, 525, A114

Lardo C. et al., 2015, A\&A, 573, A115

Lee Y.-W., Joo J.-M., Sohn Y.-J., Rey S.-C., Lee H.-C., Walker A. R., 1999, Nature, 402, 55

Long K. S., Charles P. A., Dubus G., 2002, ApJ, 569, 204

Lynden-Bell D., 1967, MNRAS, 136, 101

Lyubenova M. et al., 2013, MNRAS, 431, 3364

Mackey A. D., Gilmore G. F., 2003, MNRAS, 340, 175

McLaughlin D. E., van der Marel R. P., 2005, ApJS, 161, 304

Marino A. F., Milone A. P., Piotto G., Villanova S., Bedin L. R., Bellini A., Renzini A., 2009, A\&A, 505, 1099

Marino A. F. et al., 2011, A\&A, 532, A8

Marino A. F. et al., 2015, MNRAS, 450, 815

Marino A. F. et al., 2016, MNRAS, 459, 610

Marks M., Kroupa P., 2010, MNRAS, 406, 2000

Massari D. et al., 2014, ApJ, 795, 22

Mastrobuono-Battisti A., Perets H. B., 2013, ApJ, 779, 85

Merritt D., Valluri M., 1996, ApJ, 471, 82

Meylan G., Mayor M., Duquennoy A., Dubath P., 1995, A\&A, 303, 761

Mihos J. C., Hernquist L., 1994, ApJ, 427, 112

Milosavljević M., 2004, ApJ, 605, L13
Miocchi P., 2010, A\&A, 514, A52

Miocchi P., Capuzzo Dolcetta R., Di Matteo P., Vicari A., 2006, ApJ, 644, 940

Mucciarelli A., Lapenna E., Massari D., Ferraro F. R., Lanzoni B., 2015, ApJ, 801, 69

Noyola E., Gebhardt K., Bergmann M., 2008, ApJ, 676, 1008

Origlia L. et al., 2011, ApJ, 726, L20

Pancino E., Bellazzini M., Marinoni S., 2013, Mem. Soc. Astron. Ital., 84, 83

Perets H. B., Mastrobuono-Battisti A., 2014, ApJ, 784, L44

Pfuhl O. et al., 2011, ApJ, 741, 108

Piotto G. et al., 2015, AJ, 149, 91

Portaluri E., Corsini E. M., Morelli L., Hartmann M., Dalla Bontà E., Debattista V. P., Pizzella A., 2013, MNRAS, 433, 434

Prantzos N., Charbonnel C., 2006, A\&A, 458, 135

Renzini A. et al., 2015, MNRAS, 454, 4197

Richer H. B., Heyl J., Anderson J., Kalirai J. S., Shara M. M., Dotter A., Fahlman G. G., Rich R. M., 2013, ApJ, 771, L15

Rossa J., van der Marel R. P., Böker T., Gerssen J., Ho L. C., Rix H.-W., Shields J. C., Walcher C.-J., 2006, AJ, 132, 1074

Sarajedini A., Layden A. C., 1995, AJ, 109, 1086

Sellwood J. A., 2008, ApJ, 679, 379

Sellwood J. A., Debattista V. P., 2009, MNRAS, 398, 1279

Seth A. C., Dalcanton J. J., Hodge P. W., Debattista V. P., 2006, AJ, 132, 2539

Seth A. C., Blum R. D., Bastian N., Caldwell N., Debattista V. P., 2008, ApJ, 687, 997

Siegel M. H. et al., 2007, ApJ, 667, L57

Siegel M. H. et al., 2011, ApJ, 743, 20

Sollima A., Bellazzini M., Smart R. L., Correnti M., Pancino E., Ferraro F. R., Romano D., 2009, MNRAS, 396, 2183

Stadel J. G., 2001, PhD thesis, Univ. Washington

Tremaine S. D., Ostriker J. P., Spitzer L., Jr, 1975, ApJ, 196, 407

Turner M. L., Côté P., Ferrarese L., Jordán A., Blakeslee J. P., Mei S., Peng E. W., West M. J., 2012, ApJS, 203, 5

Valluri M., Vass I. M., Kazantzidis S., Kravtsov A. V., Bohn C. L., 2007, ApJ, 658, 731

van de Ven G., van den Bosch R. C. E., Verolme E. K., de Zeeuw P. T., 2006, A\&A, 445, 513

van den Bergh S., 1996, ApJ, 471, L31

van der Marel R. P., Anderson J., 2010, ApJ, 710, 1063

Ventura P., D’Antona F., Mazzitelli I., Gratton R., 2001, ApJ, 550, L65

Vesperini E., McMillan S. L. W., D’Antona F., D’Ercole A., 2013, MNRAS, 429, 1913

Walcher C. J. et al., 2005, ApJ, 618, 237

Walcher C. J., Böker T., Charlot S., Ho L. C., Rix H.-W., Rossa J., Shields J. C., van der Marel R. P., 2006, ApJ, 649, 692

Watkins L. L., van der Marel R. P., Bellini A., Anderson J., 2015, ApJ, 812, 149

White S. D. M., 1980, MNRAS, 191, 1P

Wrobel J. M., Greene J. E., Ho L. C., 2011, AJ, 142, 113

Yong D. et al., 2014, MNRAS, 441, 3396

This paper has been typeset from a $\mathrm{T}_{\mathrm{E}} \mathrm{X} / \mathrm{L} \mathrm{T} \mathrm{E} \mathrm{X}$ file prepared by the author. 